DESY 04-129

IFIC/04-44

FTUAM 04/17

IFT-UAM/CSIC-04-42

hep-ph/yymmddd

August 7, 2004

\title{
Theoretical predictions for the direct detection of neutralino dark matter in the NMSSM
}

\author{
D. G. Cerdeño ${ }^{a}$, C. Hugonie ${ }^{b}$, D.E. López-Fogliani ${ }^{c}$, C. Muñoz ${ }^{c}$ and A. M. Teixeira ${ }^{c}$ \\ ${ }^{a}$ II. Institut für Theoretische Physik, Universität Hamburg \\ Luruper Chaussee 149, D-22761 Hamburg, Germany \\ ${ }^{b}$ AHEP Group, Instituto de Física Corpuscular - CSIC/Universitat de València \\ Edificio Institutos de Investigación, Apartado de Correos 22085, \\ E-46071 València, Spain \\ ${ }^{c}$ Departamento de Física Teórica C-XI and Instituto de Física Teórica C-XVI, \\ Universidad Autónoma de Madrid, Cantoblanco, E-28049 Madrid, Spain
}

\begin{abstract}
We analyse the direct detection of neutralino dark matter in the framework of the Next-to-Minimal Supersymmetric Standard Model. After performing a detailed analysis of the parameter space, taking into account all the available constraints from LEPII, we compute the neutralino-nucleon cross section, and compare the results with the sensitivity of detectors. We find that sizable values for the detection cross section, within the reach of dark matter detectors, are attainable in this framework. For example, neutralino-proton cross sections compatible with the sensitivity of present experiments can be obtained due to the exchange of very light Higgses with $m_{h_{1}^{0}} \lesssim 70 \mathrm{GeV}$. Such Higgses have a significant singlet composition, thus escaping detection and being in agreement with accelerator data. The lightest neutralino in these cases exhibits a large singlino-Higgsino composition, and a mass in the range $50 \lesssim m_{\tilde{\chi}_{1}^{0}} \lesssim 100 \mathrm{GeV}$.
\end{abstract}

PACS: 12.60.Jv, 95.35.+d 


\section{Introduction}

One of the most important enigmas in physics is the problem of the dark matter in the Universe. Particle physics, and in particular extensions of the standard model (SM) offer candidates for dark matter. Among the most interesting ones are Weakly Interacting Massive Particles (WIMPs), since these can be left over from the Big Bang in sufficient number to account for a significant fraction of the observed matter density.

Since 1987, impressive experimental efforts have been carried out for the direct detection of WIMPs through elastic scattering with nuclei in a detector [1]. In fact, one of the experiments, the DAMA collaboration, reported data favouring the existence of a WIMP signal [2]. Once uncertainties on the halo model are taken into account $[2,3]$, this signal is compatible with WIMP masses smaller than 500-900 GeV and with WIMP-nucleon cross sections in the range $\sigma \approx 10^{-7}-6 \times 10^{-5} \mathrm{pb}$.

However, this result has not been confirmed by the other collaborations. In particular, CDMS Soudan [4], EDELWEISS [5] and ZEPLIN I [6] have excluded important regions of the DAMA parameter space ${ }^{1}$. In the light of these experimental results more than 20 experiments are running or in preparation around the world. For example, this is the case of GEDEON [8], which will be able to explore positively a WIMP-nucleon cross section $\sigma \gtrsim 3 \times 10^{-8} \mathrm{pb}$. CDMS Soudan will be able to test in the future $\sigma \gtrsim 2 \times 10^{-8} \mathrm{pb}$, and the very sensitive detector GENIUS [9], will be able to test a WIMP-nucleon cross section $\sigma \approx 10^{-9} \mathrm{pb}$. In fact, already planned detectors working with 1 tonne of $\mathrm{Ge} / \mathrm{Xe}$ are expected to reach cross sections as low as $10^{-10} \mathrm{pb}$ $[10]$.

Given this situation, and assuming that the dark matter is a WIMP, it is necessary to analyse the theoretical predictions for the WIMP-nucleon cross section. Obviously, the answer to this question depends on the particular WIMP considered. The leading candidate in this class of particles is the lightest neutralino, $\tilde{\chi}_{1}^{0}$, which appears in supersymmetric (SUSY) extensions of the SM [11]. The cross section for the elastic scattering of $\tilde{\chi}_{1}^{0}$ on nucleons has been examined exhaustively in the context of the Minimal Supersymmetric Standard Model (MSSM) [1]. In particular, there are regions of the parameter space of the MSSM where the neutralino-nucleon cross section is compatible with the sensitivity of present (and future) dark matter detectors.

However, it is well known that the MSSM faces a naturalness problem - the so-

\footnotetext{
${ }^{1}$ For attempts to show that DAMA and these experiments might not be in conflict, see Ref. [7].
} 
called $\mu$ problem [12] - arising from the presence of a mass term for the Higgs fields in the superpotential, $\mu H_{1} H_{2}$. The only natural values for the $\mu$ parameter are either zero or the Planck scale. The first is experimentally excluded since it leads to an unacceptable axion once the electroweak (EW) symmetry is broken, while the latter reintroduces the hierarchy problem. There exist explanations for an $\mathcal{O}\left(M_{W}\right)$ value for the $\mu$ term, although all in extended frameworks $[12,13]$.

The Next-to-Minimal Supersymmetric Standard Model (NMSSM) [14-25] provides an elegant solution to the $\mu$ problem of the MSSM via the introduction of a singlet superfield $S$. In the simplest form of the superpotential, which is scale invariant and contains the $\mathrm{SH}_{1} \mathrm{H}_{2}$ coupling, an effective $\mu$ term is generated when the scalar component of $S$ acquires a vacuum expectation value (VEV) of order the SUSY breaking scale. This effective coupling is naturally of order the EW scale if the SUSY breaking scale is not too large compared with $M_{W}$. In fact, the NMSSM is the simplest supersymmetric extension of the standard model in which the EW scale exclusively originates from the SUSY breaking scale. Another appealing feature of the NMSSM is related to the "little fine tuning problem" of the MSSM, or equivalently, the non-observation of a neutral CP-even Higgs boson at LEP II. As shown in [16], in the context of the NMSSM the latter problem becomes less severe. Although the symmetries of the NMSSM may give rise to the possibility of a cosmological domain wall problem [17], this can be avoided by the introduction of suitable non-normalisable operators [18] that do not generate dangerously large singlet tadpole diagrams [26]. These additional operators can be chosen small enough as not to alter the low energy phenomenology.

In addition to the MSSM fields, the NMSSM contains an extra CP-even and CP-odd neutral Higgs bosons, as well as one additional neutralino. These new fields mix with the corresponding MSSM ones, giving rise to a richer and more complex phenomenology [15, 19-22]. A very light neutralino may be present [19]. The upper bound on the mass of the lightest Higgs state is larger than in the MSSM [20]. Moreover, a very light Higgs boson is not experimentally excluded [21,22]. All these properties may modify the results concerning the neutralino-nucleon cross section with respect to those of the MSSM.

In fact, in comparison with the MSSM, there are only a few works in the literature studying the direct detection of the lightest neutralino in the NMSSM [23,24], as well as its relic density [25]. Thus, given the recent experimental results concerning the detection of dark matter, and in view of the appealing theoretical and phenomenological properties of the NMSSM, it is important to carry out an up-to-date analysis of the 
neutralino-nucleon cross section in this framework. Moreover, the recently published FORTRAN code NMHDECAY [21] allows a precise calculation of the particle spectrum in the NMSSM, as well as a complete check of all the available experimental constraints from LEP, thus enabling a thorough study of $\sigma$ in the allowed parameter space of the NMSSM.

The outline of the paper is as follows: In Section 2 we introduce the model, discussing in particular its Higgs potential, Higgs and neutralino mass matrices, and the parameter space. In Section 3 we examine the relevant effective Lagrangian describing the elastic $\tilde{\chi}_{1}^{0}$-nucleon scattering and its associated cross section. Section 4 is devoted to the presentation of the results for the $\tilde{\chi}_{1}^{0}$-nucleon cross section in the NMSSM, taking into account the relevant constraints on the parameter space from accelerator data. Our conclusions are given in Section 5.

\section{Overview of the NMSSM}

In this Section, we review some important features of the NMSSM. In particular, we discuss the Higgs and neutralino sectors of the model, presenting the tree-level mass matrices and mixings which are relevant for our analysis. We also discuss the theoretical and experimental constraints, and how these are reflected in the parameter space.

\subsection{Higgs scalar potential}

In addition to the MSSM Yukawa couplings for quarks and leptons, the NMSSM superpotential contains two additional terms involving the Higgs doublet superfields, $H_{1}$ and $H_{2}$, and the new superfield $S$, a singlet under the SM gauge group $S U(3)_{c} \times S U(2)_{L} \times$ $U(1)_{Y}$,

$$
W=\epsilon_{i j}\left(Y_{u} H_{2}^{j} Q^{i} u+Y_{d} H_{1}^{i} Q^{j} d+Y_{e} H_{1}^{i} L^{j} e\right)-\epsilon_{i j} \lambda S H_{1}^{i} H_{2}^{j}+\frac{1}{3} \kappa S^{3},
$$

where we take $H_{1}^{T}=\left(H_{1}^{0}, H_{1}^{-}\right), H_{2}^{T}=\left(H_{2}^{+}, H_{2}^{0}\right), i, j$ are $S U(2)$ indices, and $\epsilon_{12}=1$. In this model, the usual MSSM bilinear $\mu$ term is absent from the superpotential, and only dimensionless trilinear couplings are present in $W$. However, when the scalar component of $S$ acquires a VEV, an effective interaction $\mu H_{1} H_{2}$ is generated, with $\mu \equiv \lambda\langle S\rangle$.

As mentioned in the Introduction, the superpotential in Eq. (2.1) is scale invariant, 
and the EW scale will only appear through the soft SUSY breaking terms in $\mathcal{L}_{\text {soft }}$, which in our conventions is given by

$$
\begin{aligned}
-\mathcal{L}_{\text {soft }}= & m_{\tilde{Q}}^{2} \tilde{Q}^{*} \tilde{Q}+m_{\tilde{U}}^{2} \tilde{u}^{*} \tilde{u}+m_{\tilde{D}}^{2} \tilde{d}^{*} \tilde{d}+m_{\tilde{L}}^{2} \tilde{L}^{*} \tilde{L}+m_{\tilde{E}}^{2} \tilde{e}^{*} \tilde{e} \\
& +m_{H_{1}}^{2} H_{1}^{*} H_{1}+m_{H_{2}}^{2} H_{2}^{*} H_{2}+m_{S}^{2} S^{*} S \\
& +\epsilon_{i j}\left(A_{u} Y_{u} H_{2}^{j} \tilde{Q}^{i} \tilde{u}+A_{d} Y_{d} H_{1}^{i} \tilde{Q}^{j} \tilde{d}+A_{e} Y_{e} H_{1}^{i} \tilde{L}^{j} \tilde{e}+\text { H.c. }\right) \\
& +\left(-\epsilon_{i j} \lambda A_{\lambda} S H_{1}^{i} H_{2}^{j}+\frac{1}{3} \kappa A_{\kappa} S^{3}+\text { H.c. }\right) \\
& -\frac{1}{2}\left(M_{3} \lambda_{3} \lambda_{3}+M_{2} \lambda_{2} \lambda_{2}+M_{1} \lambda_{1} \lambda_{1}+\text { H.c. }\right) .
\end{aligned}
$$

In our subsequent analysis we assume that the soft breaking parameters are free at the EW scale. In addition to terms from $\mathcal{L}_{\text {soft }}$, the tree-level scalar Higgs potential receives the usual $D$ and $F$ term contributions:

$$
\begin{aligned}
V_{D}= & \frac{g_{1}^{2}+g_{2}^{2}}{8}\left(\left|H_{1}\right|^{2}-\left|H_{2}\right|^{2}\right)^{2}+\frac{g_{2}^{2}}{2}\left|H_{1}^{\dagger} H_{2}\right|^{2}, \\
V_{F}= & |\lambda|^{2}\left(\left|H_{1}\right|^{2}|S|^{2}+\left|H_{2}\right|^{2}|S|^{2}+\left|\epsilon_{i j} H_{1}^{i} H_{2}^{j}\right|^{2}\right)+|\kappa|^{2}|S|^{4} \\
& -\left(\epsilon_{i j} \lambda \kappa^{*} H_{1}^{i} H_{2}^{j} S^{* 2}+\text { H.c. }\right) .
\end{aligned}
$$

\subsection{Minimization of the tree level scalar potential}

Once the EW symmetry is spontaneously broken, the neutral Higgs scalars develop the following VEV's:

$$
\left\langle H_{1}^{0}\right\rangle=v_{1}, \quad\left\langle H_{2}^{0}\right\rangle=v_{2}, \quad\langle S\rangle=s .
$$

One has to ensure the absence of non-vanishing VEV's for the charged Higgs fields, which would induce the appearance of charge breaking minima. By means of an $S U(2)_{L} \times U(1)_{Y}$ transformation, one can take, without loss of generality, $\left\langle H_{2}^{+}\right\rangle=0$ while $\left\langle H_{2}^{0}\right\rangle=v_{2} \in \mathbb{R}^{+}$. The condition to have $v_{1}^{-}=\left\langle H_{1}^{-}\right\rangle=0$ as a global minimum is quite involved; still, imposing that $v_{1}^{-}=0$ is a local minimum is equivalent to requiring that the physical charged Higgses have positive mass squared.

Bringing together all the terms in Eqs. (2.2) and (2.3), we then obtain for the 
tree-level neutral Higgs potential:

$$
\begin{aligned}
\left\langle V_{\text {neutral }}^{\text {Higgs }}\right\rangle= & \frac{g_{1}^{2}+g_{2}^{2}}{8}\left(\left|v_{1}\right|^{2}-\left|v_{2}\right|^{2}\right)^{2} \\
& +|\lambda|^{2}\left(|s|^{2}\left|v_{1}\right|^{2}+|s|^{2}\left|v_{2}\right|^{2}+\left|v_{1}\right|^{2}\left|v_{2}\right|^{2}\right)+|\kappa|^{2}|s|^{4} \\
& +m_{H_{1}}^{2}\left|v_{1}\right|^{2}+m_{H_{2}}^{2}\left|v_{2}\right|^{2}+m_{S}^{2}|s|^{2} \\
& +\left(-\lambda \kappa^{*} v_{1} v_{2} s^{* 2}-\lambda A_{\lambda} s v_{1} v_{2}+\frac{1}{3} \kappa A_{\kappa} s^{3}+\text { H.c. }\right) .
\end{aligned}
$$

In the following, we assume that $\lambda, \kappa$, as well as the soft SUSY breaking terms are real. This implies the absence of explicit $\mathrm{CP}$ violation in the scalar sector. Although $v_{1}$ and $s$ can be complex parameters, the global $\mathbb{Z}_{3}$ symmetry exhibited by the superpotential implies that CP-violating extrema of $V_{\text {neutral }}^{\text {Higgs }}$ are maxima rather than minima [27]. In principle, $\lambda, \kappa$, and the trilinear soft-breaking terms, $A_{\lambda}$ and $A_{\kappa}$, in Eq. (2.5) can have both signs.

Ensuring that the tree-level potential has a minimum with respect to the phases of the VEV's directly excludes some combinations of signs for the parameters. After conducting this analysis, and given that the potential is invariant under the symmetries $\lambda, \kappa, s \rightarrow-\lambda,-\kappa,-s$ and $\lambda, v_{1} \rightarrow-\lambda,-v_{1}$, we adopt, without loss of generality, the sign convention where both $\lambda$ and $v_{1}$ are positive. We then have only positive values for $\lambda$ and $\tan \beta$, while $\kappa$ and $\mu(=\lambda s)$, as well as $A_{\lambda}$ and $A_{\kappa}$, can have both signs.

In what follows, we summarise the conditions for $\kappa, A_{\lambda}, A_{\kappa}$ and $\mu(=\lambda s)$ obtained from the minimization of the potential with respect to the phases of the VEV's. In particular, for $\kappa>0$, one can analytically show that minima of $V_{\text {neutral }}^{\text {Higgs }}$ may be obtained for the following three combinations of signs, provided that in each case the corresponding conditions are fulfilled,

(i) $\operatorname{sign}(s)=\operatorname{sign}\left(A_{\lambda}\right)=-\operatorname{sign}\left(A_{\kappa}\right)$,

which always leads to a minimum with respect to the phases.

(ii) $\operatorname{sign}(s)=-\operatorname{sign}\left(A_{\lambda}\right)=-\operatorname{sign}\left(A_{\kappa}\right)$, with $\left|A_{\kappa}\right|>3 \lambda v_{1} v_{2}\left|A_{\lambda}\right| /\left(-\left|s A_{\lambda}\right|+\kappa\left|s^{2}\right|\right)$, where the denominator has to be positive.

(iii) $\operatorname{sign}(s)=\operatorname{sign}\left(A_{\lambda}\right)=\operatorname{sign}\left(A_{\kappa}\right)$, with $\left|A_{\kappa}\right|<3 \lambda v_{1} v_{2}\left|A_{\lambda}\right| /\left(\left|s A_{\lambda}\right|+\kappa\left|s^{2}\right|\right)$.

Similarly, for $\kappa<0$, minima can only be obtained for the combination 
(iv) $\operatorname{sign}(s)=\operatorname{sign}\left(A_{\lambda}\right)=\operatorname{sign}\left(A_{\kappa}\right)$, with $\left|A_{\kappa}\right|>3 \lambda v_{1} v_{2}\left|A_{\lambda}\right| /\left(\left|s A_{\lambda}\right|-\kappa\left|s^{2}\right|\right)$, where the denominator has to be positive.

Numerically, one finds that these tree-level conditions generally hold even after the inclusion of higher order corrections.

One can derive three minimization conditions for the Higgs VEV's and use them to re-express the soft breaking Higgs masses in terms of $\lambda, \kappa, A_{\lambda}, A_{\kappa}, v_{1}, v_{2}$ and $s$ :

$$
\begin{aligned}
& m_{H_{1}}^{2}=-\lambda^{2}\left(s^{2}+v^{2} \sin ^{2} \beta\right)-\frac{1}{2} M_{Z}^{2} \cos 2 \beta+\lambda s \tan \beta\left(\kappa s+A_{\lambda}\right), \\
& m_{H_{2}}^{2}=-\lambda^{2}\left(s^{2}+v^{2} \cos ^{2} \beta\right)+\frac{1}{2} M_{Z}^{2} \cos 2 \beta+\lambda s \cot \beta\left(\kappa s+A_{\lambda}\right), \\
& m_{S}^{2}=-\lambda^{2} v^{2}-2 \kappa^{2} s^{2}+\lambda \kappa v^{2} \sin 2 \beta+\frac{\lambda A_{\lambda} v^{2}}{2 s} \sin 2 \beta-\kappa A_{\kappa} s,
\end{aligned}
$$

where $v^{2}=v_{1}^{2}+v_{2}^{2}=2 M_{W}^{2} / g_{2}^{2}$ and $\tan \beta=v_{2} / v_{1}$.

\subsection{Higgs boson mass matrices}

Subsequent to EW symmetry breaking, and after rotating away the CP-odd would-be Goldstone boson, we are left with five neutral Higgs states and two charged Higgs states. Assuming

$$
H_{1}^{0} \equiv v_{1}+\frac{H_{1 R}+i H_{1 I}}{\sqrt{2}}, \quad H_{2}^{0} \equiv v_{2}+\frac{H_{2 R}+i H_{2 I}}{\sqrt{2}}, \quad S \equiv s+\frac{S_{R}+i S_{I}}{\sqrt{2}},
$$

among the neutral Higgses we find three CP-even states - $H_{1 R}, H_{2 R}, S_{R}$ and two CP-odd components, $A^{0}$, $S_{I}$, with $A^{0}$ related to the original fields as $H_{1(2) I}=\sin \beta(\cos \beta) A^{0}$. Using the minimization conditions above, the tree-level mass matrix for the neutral Higgs bosons can be easily obtained. Since we have made the assumption that there is no $\mathrm{CP}$-violation on the Higgs sector, CP-even and CP-odd states do not mix, and the corresponding mass matrices can be written in the respective basis, $H^{0}=\left(H_{1 R}, H_{2 R}, S_{R}\right)$ 
and $P^{0}=\left(A^{0}, S_{I}\right)$. For the CP-even states, we have

$$
\begin{aligned}
& \mathcal{M}_{S, 11}^{2}=M_{Z}^{2} \cos ^{2} \beta+\lambda s \tan \beta\left(A_{\lambda}+\kappa s\right) \\
& \mathcal{M}_{S, 22}^{2}=M_{Z}^{2} \sin ^{2} \beta+\lambda s \cot \beta\left(A_{\lambda}+\kappa s\right) \\
& \mathcal{M}_{S, 33}^{2}=4 \kappa^{2} s^{2}+\kappa A_{\kappa} s+\frac{\lambda}{s} A_{\lambda} v_{1} v_{2} \\
& \mathcal{M}_{S, 12}^{2}=\left(\lambda^{2} v^{2}-\frac{M_{Z}^{2}}{2}\right) \sin 2 \beta-\lambda s\left(A_{\lambda}+\kappa s\right) \\
& \mathcal{M}_{S, 13}^{2}=2 \lambda^{2} v_{1} s-\lambda v_{2}\left(A_{\lambda}+2 \kappa s\right) \\
& \mathcal{M}_{S, 23}^{2}=2 \lambda^{2} v_{2} s-\lambda v_{1}\left(A_{\lambda}+2 \kappa s\right) .
\end{aligned}
$$

The CP-even Higgs interaction and physical eigenstates are related by the transformation

$$
h_{a}^{0}=S_{a b} H_{b}^{0},
$$

where $S$ is the unitary matrix that diagonalises the above symmetric mass matrix, $a, b=1,2,3$, and the physical eigenstates are ordered $\operatorname{as}^{2} m_{h_{1}^{0}} \lesssim m_{h_{2}^{0}} \lesssim m_{h_{3}^{0}}$. In the pseudoscalar sector, after rewriting the CP-odd mass terms in the $P^{0}$ basis, the corresponding (symmetric) mass matrix reads

$$
\begin{aligned}
& \mathcal{M}_{P, 11}^{2}=\frac{2 \lambda s}{\sin 2 \beta}\left(\kappa s+A_{\lambda}\right) \\
& \mathcal{M}_{P, 22}^{2}=\lambda\left(2 \kappa+\frac{A_{\lambda}}{2 s}\right) v^{2} \sin 2 \beta-3 \kappa A_{\kappa} s \\
& \mathcal{M}_{P, 12}^{2}=\lambda v\left(A_{\lambda}-2 \kappa s\right),
\end{aligned}
$$

and the relation between physical and interaction eigenstates is given by

$$
a_{i}^{0}=P_{i j} P_{j}^{0}
$$

Regarding the charged Higgs mass, at the tree level it is given by

$$
m_{H^{ \pm}}^{2}=M_{W}^{2}-\lambda^{2} v^{2}+\lambda\left(A_{\lambda}+\kappa s\right) \frac{2 s}{\sin 2 \beta} .
$$

\subsection{Neutralino mass matrix}

When compared to the MSSM case, the structure of chargino and squark mass terms is essentially unaffected, provided that one uses $\mu=\lambda s$. However, in the neutralino

\footnotetext{
${ }^{2}$ Throughout the paper we always adopt the convention $m_{i} \lesssim m_{j}$ for $i<j$.
} 
sector, the situation is more involved, since the fermionic component of $S$ mixes with the neutral Higgsinos, giving rise to a fifth neutralino state. In the weak interaction basis defined by $\Psi^{0^{T}} \equiv\left(\tilde{B}^{0}=-i \lambda^{\prime}, \tilde{W}_{3}^{0}=-i \lambda_{3}, \tilde{H}_{1}^{0}, \tilde{H}_{2}^{0}, \tilde{S}\right)$, the neutralino mass terms in the Lagrangian are

$$
\mathcal{L}_{\text {mass }}^{\tilde{\chi}^{0}}=-\frac{1}{2}\left(\Psi^{0}\right)^{T} \mathcal{M}_{\tilde{\chi}^{0}} \Psi^{0}+\text { H.c. },
$$

with $\mathcal{M}_{\tilde{\chi}^{0}}$ a $5 \times 5$ matrix,

$$
\mathcal{M}_{\tilde{\chi}^{0}}=\left(\begin{array}{ccccc}
M_{1} & 0 & -M_{Z} \sin \theta_{W} \cos \beta & M_{Z} \sin \theta_{W} \sin \beta & 0 \\
0 & M_{2} & M_{Z} \cos \theta_{W} \cos \beta & -M_{Z} \cos \theta_{W} \sin \beta & 0 \\
-M_{Z} \sin \theta_{W} \cos \beta & M_{Z} \cos \theta_{W} \cos \beta & 0 & -\lambda s & -\lambda v_{2} \\
M_{Z} \sin \theta_{W} \sin \beta & -M_{Z} \cos \theta_{W} \sin \beta & -\lambda s & 0 & -\lambda v_{1} \\
0 & 0 & -\lambda v_{2} & -\lambda v_{1} & 2 \kappa s
\end{array}\right) .
$$

The above matrix can be diagonalised by means of a unitary matrix $N$,

$$
N^{*} \mathcal{M}_{\tilde{\chi}^{0}} N^{-1}=\operatorname{diag}\left(m_{\tilde{\chi}_{1}^{0}}, m_{\tilde{\chi}_{2}^{0}}, m_{\tilde{\chi}_{3}^{0}}, m_{\tilde{\chi}_{4}^{0}}, m_{\tilde{\chi}_{5}^{0}}\right)
$$

where $m_{\tilde{\chi}_{1}^{0}}$ is the lightest neutralino mass. Under the above assumptions, the lightest neutralino can be expressed as the combination

$$
\tilde{\chi}_{1}^{0}=N_{11} \tilde{B}^{0}+N_{12} \tilde{W}_{3}^{0}+N_{13} \tilde{H}_{1}^{0}+N_{14} \tilde{H}_{2}^{0}+N_{15} \tilde{S}
$$

In the following, neutralinos with $N_{13}^{2}+N_{14}^{2}>0.9$, or $N_{15}^{2}>0.9$, will be referred to as Higgsino- or singlino-like, respectively.

\subsection{NMSSM parameter space}

At the weak scale, the free parameters in the Higgs sector are (at tree level): $\lambda, \kappa$, $m_{H_{1}}^{2}, m_{H_{2}}^{2}, m_{S}^{2}, A_{\lambda}$ and $A_{\kappa}$. Using the three minimization conditions of the Higgs potential (including the dominant one- and two-loop corrections), one can eliminate the soft Higgs masses in favour of $M_{Z}, \tan \beta$ and $\mu$. We thus consider as independent parameters the following set of variables

$$
\lambda, \kappa, \tan \beta, \mu, A_{\lambda}, A_{\kappa} .
$$

In our study, the soft scalar masses as well as the soft gaugino soft masses $M_{i}$ are free parameters at the EW scale. We scanned over the parameter space using the program NMHDECAY [21] and in what follows we overview the most relevant aspects of the analysis. 
For each point in the parameter space, one requires the absence of Landau singularities for $\lambda, \kappa, Y_{t}$ and $Y_{b}$ below the GUT scale. For $m_{t}^{\text {pole }}=175 \mathrm{GeV}$, this translates into $\lambda \lesssim 0.75,|\kappa| \lesssim 0.65$, and $1.7 \lesssim \tan \beta \lesssim 54$. In addition one verifies that the physical minimum is a true one, in other words, that it is deeper than the local unphysical minima with $\left\langle H_{1,2}^{0}\right\rangle=0$ and/or $\langle S\rangle=0$.

One then computes the scalar, pseudo-scalar and charged Higgs masses and mixings, taking into account 1- and 2-loop radiative corrections. The dominant 1-loop corrections to the Higgs masses originate from top, stop, bottom and sbottom loops, and the corresponding corrections to $m_{h_{1}^{0}}^{2}$ are of $\mathcal{O}\left(Y_{t, b}^{4}\right)$. Pure electroweak contributions of $\mathcal{O}\left(g^{2}\right)$ are also taken into account. Regarding 2-loop corrections to the effective potential, the dominant ones are associated with top-stop loops, and only the leading (double) logarithms are included. The chargino and neutralino masses and mixings are computed and the couplings of the scalar and pseudoscalar Higgs to charginos and neutralinos are calculated.

Finally, all available experimental constraints from LEP are checked:

1) In the neutralino sector, we check that the lightest neutralino does not contribute excessively to the invisible width of the $Z$ boson $\left(\Gamma\left(Z \rightarrow \tilde{\chi}_{1}^{0} \tilde{\chi}_{1}^{0}\right)<1.76 \mathrm{MeV}[28]\right)$ if $m_{\tilde{\chi}_{1}^{0}}<M_{Z} / 2$, and that $\sigma\left(e^{+} e^{-} \rightarrow \tilde{\chi}_{1}^{0} \tilde{\chi}_{i}^{0}\right)<10^{-2}$ pb if $m_{\tilde{\chi}_{1}^{0}}+m_{\tilde{\chi}_{i}^{0}}<209 \mathrm{GeV}(i>1)$ and $\sigma\left(e^{+} e^{-} \rightarrow \tilde{\chi}_{i}^{0} \tilde{\chi}_{j}^{0}\right)<10^{-1} \mathrm{pb}$ if $m_{\tilde{\chi}_{i}^{0}}+m_{\tilde{\chi}_{j}^{0}}<209 \mathrm{GeV}(i, j>1)$ [29].

2) In the chargino sector, we verify that the lightest chargino is not too light $\left(m_{\tilde{\chi}_{1}^{+}}>\right.$ $103.5 \mathrm{GeV}[30])$. This leads to a lower bound on $|\mu| \gtrsim 100 \mathrm{GeV}$.

3) In the charged Higgs sector, we impose $m_{H^{+}}>78.6 \mathrm{GeV}$ [31].

4) In the neutral Higgs sector, we check the constraints on the production rates (reduced couplings $) \times$ branching ratios versus the masses, for all the CP-even states $h^{0}$ and CPodd states $a^{0}$, in all the channels studied at LEP [32]: $e^{+} e^{-} \rightarrow h^{0} Z$, independent of the $h^{0}$ decay mode (IHDM); $e^{+} e^{-} \rightarrow h^{0} Z$, dependent on the $h^{0}$ decay mode (DHDM), with the Higgs decaying via $h^{0} \rightarrow b \bar{b}, h^{0} \rightarrow \tau^{+} \tau^{-}, h^{0} \rightarrow 2$ jets $h^{0} \rightarrow \gamma \gamma$ and $h^{0} \rightarrow$ invisible; associated production modes (APM), $e^{+} e^{-} \rightarrow h^{0} a^{0}$, with $h^{0} a^{0} \rightarrow 4 b^{\prime}$ s, $h^{0} a^{0} \rightarrow 4 \tau^{\prime}$ s and $h^{0} a^{0} \rightarrow a^{0} a^{0} a^{0} \rightarrow 6 b$ 's (see [21] for a detailed discussion).

It is worth noticing here that other available experimental bounds such as e.g. the $b \rightarrow s \gamma$ branching ratio, and the current upper limit on the decay $B_{s} \rightarrow \mu^{+} \mu^{-}$, might also produce relevant constraints on the parameter space. The presence of light Higgs states, in particular that of very light pseudoscalars, might translate into potentially large contributions to these processes. This issue has begun to be addressed in [33] 
for the large $\tan \beta$ regime. For instance, the branching ratio $B_{s} \rightarrow \mu^{+} \mu^{-}$scales as $\tan ^{6} \beta / m_{a^{0}}^{4}$, thus producing important constraints [34] in non-universal MSSM scenarios associated with light pseudoscalar Higgs, which favour large neutralino-nucleon cross sections. These constraints become specially relevant in the large $\tan \beta$ regime $(\tan \beta \gtrsim 35)$. However, notice, that although in the NMSSM scalar and pseudoscalar Higgs can indeed be very light, large neutralino-nucleon cross sections are in general obtained for the low $\tan \beta$ regime, as we will discuss in the next sections. It is important then to carry out a detailed analysis of these processes. In particular, $b, K$, and $B$ decays, together with the muon anomalous magnetic moment, may play an important role in further constraining the NMSSM parameter space. Such a study will be considered in a forthcoming publication [35].

\section{Neutralino-nucleon cross section}

The most general supersymmetric low-energy effective four-fermion Lagrangian that describes the elastic scattering of the lightest neutralino with the nucleon is given by $[36,37]$

$$
\begin{aligned}
\mathcal{L}_{\text {eff }}= & \overline{\tilde{\chi}}_{1}^{0} \gamma^{\mu} \gamma_{5} \tilde{\chi}_{1}^{0} \bar{q}_{i} \gamma_{\mu}\left(\alpha_{1 i}+\alpha_{2 i} \gamma_{5}\right) q_{i}+\alpha_{3 i} \overline{\tilde{\chi}}_{1}^{0} \tilde{\chi}_{1}^{0} \bar{q}_{i} q_{i} \\
& +\alpha_{4 i} \overline{\tilde{\chi}}_{1}^{0} \gamma_{5} \tilde{\chi}_{1}^{0} \bar{q}_{i} \gamma_{5} q_{i}+\alpha_{5 i} \overline{\tilde{\chi}}_{1}^{0} \tilde{\chi}_{1}^{0} \bar{q}_{i} \gamma_{5} q_{i}+\alpha_{6 i} \overline{\tilde{\chi}}_{1}^{0} \gamma_{5} \tilde{\chi}_{1}^{0} \bar{q}_{i} q_{i},
\end{aligned}
$$

where $i=1,2$ denotes up- and down-type quarks, and the Lagrangian is summed over the three quark generations. In the absence of CP-violating phases, the terms proportional to $\alpha_{5}$ and $\alpha_{6}$ vanish. Moreover, those associated with $\alpha_{1}$ and $\alpha_{4}$ (as well as $\alpha_{5}$ and $\alpha_{6}$, should these be present) are velocity-dependent, and can be safely neglected for our present purposes. The cross section associated with the spin-dependent coefficient $\left(\alpha_{2}\right)$ is only non-zero if the target nucleus has a non-vanishing spin and, contrary to case of the scalar (spin-independent) term, adds incoherently. For the case of heavy targets, as those used in the experiments mentioned in the Introduction, the scalar

cross section associated with $\alpha_{3}$ is in general substantially larger, and henceforth we shall focus our discussion on the latter.

We begin by decomposing $\alpha_{3 i}$ into two terms, one arising from squark $s$-channel exchange and the other from the $t$-channel, neutral Higgs mediated interaction (Fig. 1 


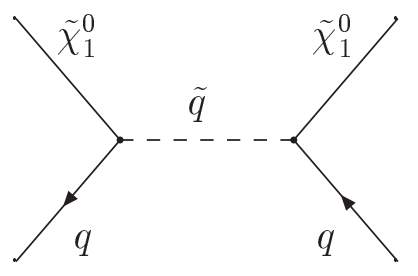

(a)

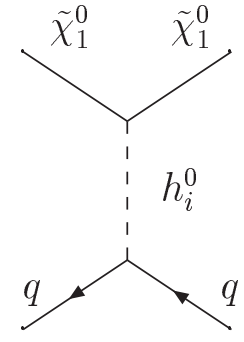

(b)

Figure 1: Feynman diagrams contributing to the neutralino-nucleon scalar cross section: (a) squark exchange and (b) scalar Higgs exchange.

(a) and (b), respectively). One obtains ${ }^{3}$

$$
\begin{aligned}
\alpha_{3 i}^{\tilde{q}} & =-\sum_{X=1}^{2} \frac{1}{4\left(m_{X i}^{2}-m_{\tilde{\chi}_{1}^{0}}^{2}\right)} \operatorname{Re}\left[\left(C_{R}^{X i}\right)\left(C_{L}^{X i}\right)^{*}\right], \\
\alpha_{3 i}^{h} & =\sum_{a=1}^{3} \frac{1}{m_{h_{a}^{0}}^{2}} C_{Y}^{i} \operatorname{Re}\left[C_{H L}^{a}\right],
\end{aligned}
$$

where $X=1,2$ denotes the squark mass eigenstates and $a=1,2,3$ refers to the scalar Higgs mass eigenstates. The relevant NMSSM couplings for the neutralinosquark-quark $\left(C_{L, R}^{X i}\right)$, neutralino-neutralino-Higgs $\left(C_{H L, R}^{a}\right)$ and Higgs-quark-quark $\left(C_{Y}^{i}\right)$ interactions are given in Appendix A.

The term $\alpha_{3 i}^{\tilde{q}}$ is formally identical to the MSSM case, differing only in the new neutralino mixings stemming from the presence of a fifth component. In particular, in regions of the NMSSM parameter space where the singlino component dominates the lightest neutralino state, there will be a significant reduction in the Bino- and Wino-squark-quark couplings, and hence in $\alpha_{3 i}^{\tilde{q}}$.

Regarding the Higgs mediated interaction term $\left(\alpha_{3 i}^{h}\right)$, the situation is slightly more involved since both vertices and the exchanged Higgs scalar significantly reflect the new features of the NMSSM. First, let us recall that in regions of the parameter space where the lightest Higgs boson has a sizable singlet component, the Higgs-quark-quark coupling might be substantially reduced. Regarding the Higgs- $\tilde{\chi}_{1}^{0}-\tilde{\chi}_{1}^{0}$ interaction, in

\footnotetext{
${ }^{3}$ When compared to the analogous expression of Ref. [24], we find some discrepancies in $\alpha_{3 i}^{h}$, namely a missing singlet-Higgsino-Higgsino term (proportional to $\lambda$ ), and the presence of an additional overall weak coupling constant, $g$.
} 
addition to a new component in the lightest $\tilde{\chi}^{0}$ state, the most important alteration emerges from the presence of new terms, proportional to $\lambda$ and $\kappa$ (cf. Appendix A). Nevertheless, and as already mentioned, light Higgs bosons can be experimentally allowed in the context of the NMSSM. Should this occur, and if these states are not pure singlets (thus displaying a non-vanishing coupling to matter) the exchange of light Higgs scalars in the $t$-channel might provide a considerable enhancement to the neutralino-nucleon cross section.

It is worth mentioning that an enhancement of $\alpha_{3 i}^{h}$ with respect to $\alpha_{3 i}^{\tilde{q}}$ is not an effect unique to the NMSSM. In fact, it has been already noticed that in the MSSM, and once the mSUGRA inspired universality for the soft scalar and gaugino masses is abandoned, the cross section associated with the channels involving scalar Higgs exchange can be substantially enhanced. Similar to what will occur in the present model, the MSSM $t$-channel contributions become larger once the Higgsino components of $\tilde{\chi}_{1}^{0}$ are augmented and/or the Higgs masses are reduced [39] (e.g. via non-universal soft masses at the GUT scale [40]).

The scalar interaction term contributes to the $\tilde{\chi}_{1}^{0}$-Nucleon cross section as

$$
\sigma_{3 N}=\frac{4 m_{r}^{2}}{\pi} f_{N}^{2}
$$

where $m_{r}$ is the Nucleon- $\tilde{\chi}_{1}^{0}$ reduced mass, $m_{r}=m_{N} m_{\tilde{\chi}_{1}^{0}} /\left(m_{N}+m_{\tilde{\chi}_{1}^{0}}\right)$, and

$$
\frac{f_{N}}{m_{N}}=\sum_{q=u, d, s} f_{T q}^{(N)} \frac{\alpha_{3 q}}{m_{q}}+\frac{2}{27} f_{T G}^{(N)} \sum_{q=c, b, t} \frac{\alpha_{3 q}}{m_{q}} .
$$

In the above, $m_{q}$ is the quark mass, and the parameters $f_{T q}^{(N)}$ are defined as $\left\langle N\left|m_{q} \bar{q} q\right| N\right\rangle$ $=m_{N} f_{T q}^{(N)} \cdot f_{T G}^{(N)}$ can be derived from $f_{T q}^{(N)}$ as $f_{T G}^{(N)}=1-\sum_{q=u, d, s} f_{T q}^{(N)}$. Following [38], we take the following values for the hadronic matrix elements:

$$
\begin{array}{lll}
f_{T u}^{(p)}=0.020 \pm 0.004, & f_{T d}^{(p)}=0.026 \pm 0.005, & f_{T s}^{(p)}=0.118 \pm 0.062, \\
f_{T u}^{(n)}=0.014 \pm 0.003, & f_{T d}^{(n)}=0.036 \pm 0.008, & f_{T s}^{(n)}=0.118 \pm 0.062 .
\end{array}
$$

In the numerical analysis of the next section we will use the central values of the above matrix elements. Notice that $f_{T s}^{(n)}=f_{T s}^{(p)}$ and both are much larger than $f_{T q}$ for $u$ and $d$ quarks, and therefore $f_{p}$ and $f_{n}$ are basically equal. Thus we will focus on the neutralino-proton cross section,

$$
\sigma_{3 p} \equiv \sigma_{\tilde{\chi}_{1}^{0}-p}=\frac{4 m_{r}^{2}}{\pi} f_{p}^{2}
$$

with $m_{r}=m_{p} m_{\tilde{\chi}_{1}^{0}} /\left(m_{p}+m_{\tilde{\chi}_{1}^{0}}\right) \sim m_{p}$. 


\section{Results and discussion}

In this Section the viability of the detection of the lightest NMSSM neutralino as a dark matter candidate, will be studied. In particular, we will compute the theoretical predictions for the direct detection of neutralinos through their elastic scattering with nucleons inside a detector. In our computation we will take into account relevant constraints on the parameter space from accelerator data. On the other hand, given the complexity of the computation of the relic neutralino density, we prefer to consider in a forthcoming publication [41] the constraints arising from reproducing the WMAP data [42], $0.094 \lesssim \Omega_{\mathrm{DM}} h^{2} \lesssim 0.129$, on our relevant parameter space for the cross section. In particular, we will see in this section that light pseudoscalars are present in interesting regions of the parameter space, and this might translate into large contributions to the annihilation cross section, implying a reduction in the associated relic density. Therefore we may expect the WMAP lower bound to play an important role in those points with a very large neutralino-nucleon cross section. For this study we will use the general analysis of the relic neutralino density, including coannihilations, that has been carried out for the NMSSM in [43].

As discussed in the Introduction, many experiments for the direct detection of dark matter are running or in preparation. Thus, in our analysis, we will be particularly interested in the various NMSSM scenarios which might potentially lead to values of $\sigma_{\tilde{\chi}_{1}^{0}-p}$ in the sensitivity range of those detectors.

Although the free parameters in our model have already been presented in Section 2.5, it is worth recalling that the Higgs and neutralino sectors of the theory are specified by

$$
\lambda, \quad \kappa, \quad \mu(=\lambda s), \quad \tan \beta, \quad A_{\lambda}, \quad A_{\kappa}, \quad M_{1}, \quad M_{2} .
$$

As aforementioned, we take these parameters to be free at the EW scale. Based on an argument of simplicity ${ }^{4}$, the low-energy squark masses and trilinear couplings, which appear in the computation of the neutralino-nucleon cross section, are taken to be degenerate $^{5}$. Unless otherwise stated, the common SUSY scale will be $M_{\text {SUSY }}=1 \mathrm{TeV}$.

\footnotetext{
${ }^{4}$ Since in our analysis of the neutralino-nucleon cross section the detection channels mediated by Higgs scalars will be enhanced with respect to those mediated by squarks, the sensitivity of the results to variations of the squark parameters will be very small.

${ }^{5}$ Regarding the stop mass matrix we will work in the maximal-mixing regime, where the off-diagonal term takes the form $m_{t} X_{t}=m_{t} \sqrt{6} M_{\mathrm{SUSY}}$. Departures from this case would not affect significantly the theoretical predictions for the neutralino-nucleon cross section.
} 
Having free squark and slepton soft parameters at the EW scale allows us to ensure that in our analysis the lightest SUSY particle is indeed the $\tilde{\chi}_{1}^{0}$. Also led by arguments of naturalness, we shall take a lower bound for $\lambda, \lambda_{\min } \sim \mu / s_{\max }$. Thus, taking the conservative range $s \lesssim 10 \mathrm{TeV}$, this translates into $\lambda_{\min } \sim \mu(\mathrm{GeV}) \times 10^{-4}$.

We begin our analysis by taking values for the soft gaugino masses that mimic at low scale the results from a hypothetical unified value at the GUT scale. Consequently, we will choose $M_{2}=1 \mathrm{TeV}$ and $M_{1}=500 \mathrm{GeV}$. For the gluino mass, the value $M_{3}=3 \mathrm{TeV}$ will be taken. Later on we will address variations of these values. In the following we take $|\mu| \geq 110 \mathrm{GeV}$, since in most cases this allows to safely avoid the LEP bound on the lightest chargino mass. Throughout this Section, we shall consider several choices for the values of $A_{\lambda}, A_{\kappa}, \mu$ and $\tan \beta$, and for each case, we will study the associated phenomenology.

In order to simultaneously analyse the dark matter predictions and understand the effect of the experimental constraints on the parameter space of the NMSSM, it is very illustrative to begin our study in the plane generated by the Higgs couplings in the superpotential, $\lambda$ and $\kappa$. In Section 2.2 we commented on the conditions to be applied to each of the sign combinations of the parameters, which arise from ensuring that the tree-level potential has a minimum with respect to the phases of the VEV's. In the following it will be clarifying to discuss each case separately. Let us first consider the cases associated with positive values of $\kappa$.

\section{1 $\mu A_{\kappa}<0$ and $\mu A_{\lambda}>0(\kappa>0)$}

As a first choice, we will consider the two cases where $\mu A_{\kappa}<0$ and $\mu A_{\lambda}>0$, namely those with $\mu, A_{\lambda},-A_{\kappa}>0$ and $\mu, A_{\lambda},-A_{\kappa}<0$.

In both cases, part of the parameter space can be excluded due to the occurrence of tachyons in the CP-even Higgs sector. Namely, it is easy to see from the expression of the CP-even Higgs matrix (2.8) that the off-diagonal terms $\left|\mathcal{M}_{S, 13}^{2}\right|$ or $\left|\mathcal{M}_{S, 23}^{2}\right|$ can become significantly bigger than $\mathcal{M}_{S, 33}^{2}$, thus leading to the appearance of a negative eigenvalue. This will typically happen for moderate to large values of $\lambda$ and small $\kappa$, for which $m_{h_{1}^{0}}$ is small. Large values of $\left|A_{\kappa}\right|$ and $\tan \beta$ lead to an increase of the tachyonic region, as we will later see. On the other hand, the eigenvalues of the CP-odd Higgs mass matrix are never negative. The CP-odd Higgs masses also decrease for large $\lambda$ and small $\kappa$, but their minimum value is bounded by the appearance of tachyons in the CP-even sector. 

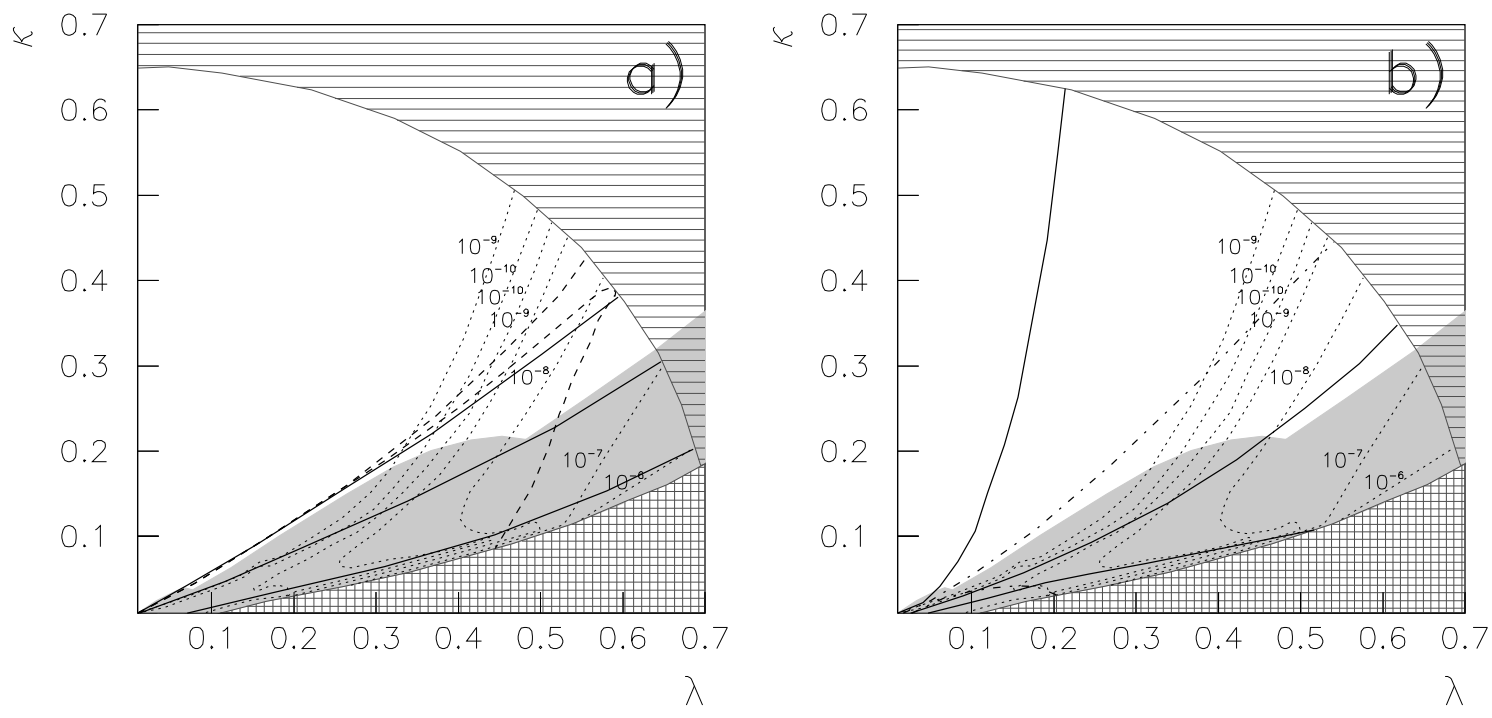

Figure 2: $(\lambda, \kappa)$ parameter space for $\tan \beta=3, A_{\lambda}=200 \mathrm{GeV}, A_{\kappa}=-50 \mathrm{GeV}$ and $\mu=110 \mathrm{GeV}$. In both cases, the ruled area represents points which are excluded due to the occurrence of a Landau pole. The grided area is excluded because of the appearance of tachyons. The grey area is associated to those points that do not satisfy the LEP constraints or where (at least) the LEP bound on direct neutralino production is violated. Dotted lines in the experimentally accepted region represent contours of scalar neutralino-proton cross section $\sigma_{\tilde{\chi}_{1}^{0}-p}$. In (a), from top to bottom, solid lines indicate different values of lightest Higgs scalar mass, $m_{h_{1}^{0}}=114,75,25 \mathrm{GeV}$, and dashed lines separate the regions where the lightest scalar Higgs has a singlet composition given by $S_{13}^{2}=0.1,0.9$. In (b), from top to bottom, solid lines are associated with different values of the lightest neutralino mass, $m_{\tilde{\chi}_{1}^{0}}=100,75,50 \mathrm{GeV}$, while dot-dashed lines reflect the singlino composition of the lightest neutralino, $N_{15}^{2}=0.1,0.9$.

The $(\lambda, \kappa)$ parameter space is shown in Fig. 2 for an example with $\tan \beta=3$, $A_{\lambda}=200 \mathrm{GeV}, A_{\kappa}=-50 \mathrm{GeV}$ and $\mu=110 \mathrm{GeV}$. The points which are excluded due to the occurrence of a Landau pole are indicated, as well as those not fulfilling the experimental constraints. According to the discussion above, the tachyons appearing in the lower right corner are due to the CP-even Higgs sector. It is worth remarking that in these cases, due to the smallness of the lightest CP-even Higgs mass, the experimental constraints (see Section 2.5) from $e^{+} e^{-} \rightarrow h^{0} Z$, both IHDM and DHDM ( $h^{0} \rightarrow b \bar{b}, h^{0} \rightarrow \tau^{+} \tau^{-}$, and $h^{0} \rightarrow 2$ jets), become very important and typically exclude the regions in the vicinity of those excluded by tachyons. 
Dashed lines in Fig. 2a indicate the singlet composition of the lightest scalar Higgs. Singlet-like Higgses can be found for small values of $\kappa$, whereas doublet-like Higgses appear for large $\kappa$. This can be qualitatively understood from the expression of the corresponding mass matrix (2.14). In particular, the diagonal term $\mathcal{M}_{S, 33}^{2}$ becomes very small when $\kappa$ decreases. Interestingly, when the singlet composition is significant, the reduced coupling can be smaller and thus Higgses with $m_{h_{1}^{0}} \lesssim 114 \mathrm{GeV}$ can escape detection and be in agreement with experimental data. This opens a new window in the allowed parameter space, characteristic of the NMSSM, which can have relevant consequences for dark matter detection as we will discuss below.

In Fig. 2b the same case is represented, but emphasizing the information on the neutralino properties. The singlino composition of the lightest neutralino is shown with dot-dashed lines, while solid lines correspond to different values of its mass. As one would expect from the structure of the neutralino mass matrix (2.14), for small $\kappa$, the lightest neutralino is essentially a singlino, with a small mass which can be approximated as $m_{\tilde{\chi}_{1}^{0}} \sim 2 \mu \kappa / \lambda$. In the present case, singlino-like neutralinos appear for $\kappa \lesssim 0.04$ and $\lambda \lesssim 0.2$, whereas heavier, Higgsino-like, neutralinos (due to our choice of input values with $\mu<M_{1}$ ) populate the rest of the parameter space. Regions with small masses of the neutralino may be excluded due to the bound on direct neutralino production, which becomes quite severe for light Higgsino-like neutralinos.

In both figures, the different values of the neutralino-nucleon cross section are represented with dotted lines. As already commented in Section 3, the cross section increases in those regions with a light CP-even Higgs, as long as it is not a pure singlet. This behaviour is clearly illustrated in these figures, which feature very large values for $\sigma_{\tilde{\chi}_{1}^{0}-p}$ in the vicinity of the areas where the lightest Higgs becomes tachyonic. On the other hand far from these regions the cross section stabilizes at $10^{-8} \mathrm{pb}>\sigma_{\tilde{\chi}_{1}^{0}-p}>10^{-9} \mathrm{pb}$.

In order to illustrate this point in more detail, we have represented in Fig. 3 the resulting $\sigma_{\tilde{\chi}_{1}^{0}-p}$ versus the lightest Higgs mass and the neutralino mass. Black dots fulfil all the experimental constraints, whereas grey dots are those experimentally excluded (we do not plot those regions ruled out due to theoretical arguments, such as the occurrence of a Landau pole). The sensitivities of present and projected dark matter experiments are also depicted as a function of $m_{\tilde{\chi}_{1}^{0}}$ for comparison. The small area bounded by dotted lines is allowed by the DAMA experiment in the simple case of an isothermal spherical halo model. The larger area also bounded by dotted lines represents the DAMA region when uncertainties to this simple model are taken into account. For the other experiments in the figure only the spherical halo has been 

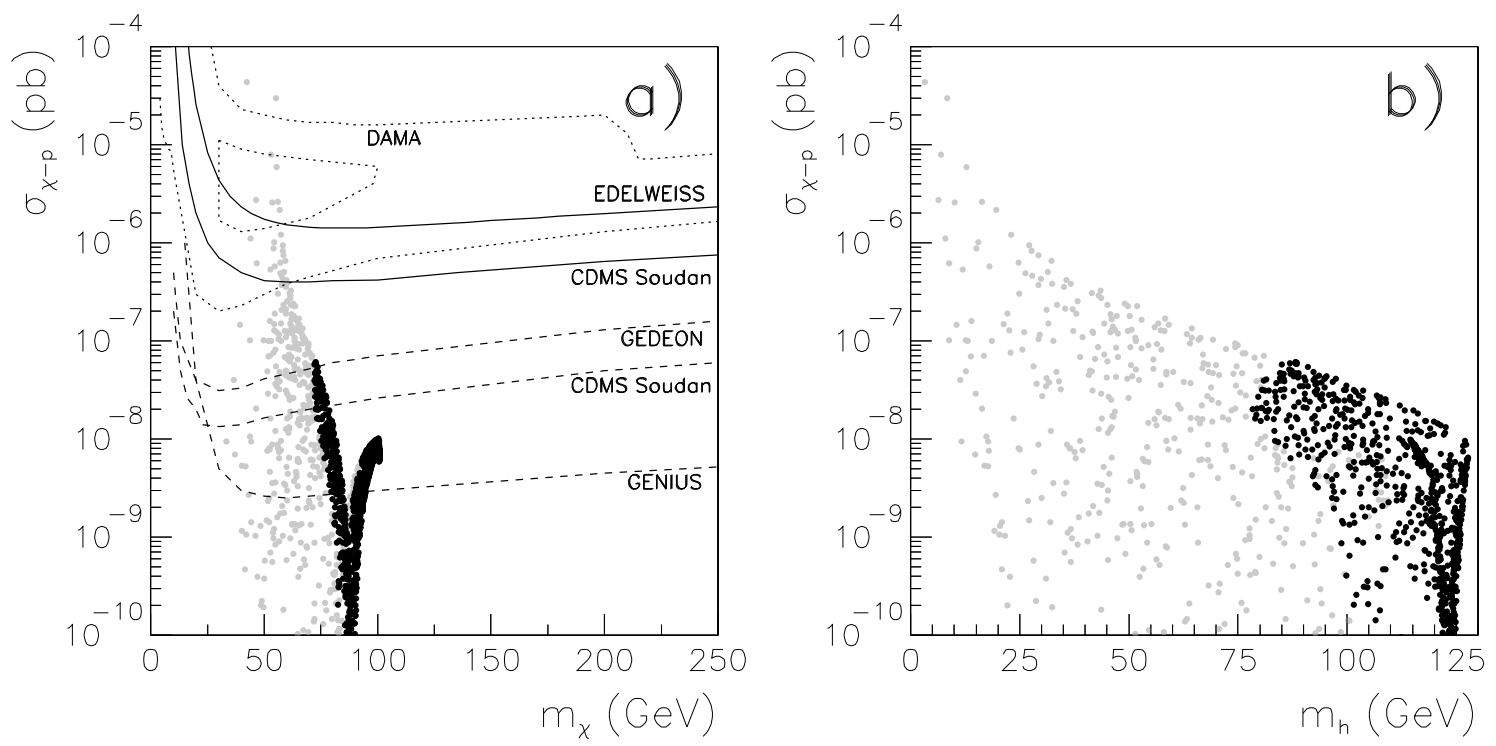

Figure 3: Scatter plot of the scalar neutralino-nucleon cross section, $\sigma_{\tilde{\chi}_{1}^{0}-p}$, as a function of (a) the neutralino mass, $m_{\tilde{\chi}_{1}^{0}}$, and (b) the lightest scalar Higgs mass, $m_{h_{1}^{0}}$, for $A_{\lambda}=200$ $\mathrm{GeV}, \mu=110 \mathrm{GeV}, A_{\kappa}=-50 \mathrm{GeV}$, and $\tan \beta=3$. Black dots correspond to points fulfilling all the experimental constraints, whereas grey dots represent those excluded. In (a) the sensitivities of present and projected experiments are also depicted with solid and dashed lines, respectively. The large (small) area bounded by dotted lines is allowed by the DAMA experiment when astrophysical uncertainties are (are not) taken into account.

considered in their analyses. In particular, the (upper) areas bounded by solid lines are excluded by EDELWEISS ${ }^{6}$ and CDMS Soudan. Finally, the dashed lines represent the sensitivities of the projected GEDEON, CDMS Soudan, and GENIUS experiments.

Very large values for the cross section could in principle be obtained. However, these are associated to very light Higgses and are therefore subject to the strong constraints on $e^{+} e^{-} \rightarrow h^{0} Z$ discussed above. Once every constraint is taken into account, points with $\sigma_{\tilde{\chi}_{1}^{0}-p} \lesssim 10^{-7} \mathrm{pb}$ appear, which correspond to light scalar Higgses with $m_{h_{1}^{0}} \gtrsim 75 \mathrm{GeV}$, surviving the experimental constraints due to their important singlet character, $S_{13}^{2} \gtrsim 0.85$. This is a clear consequence of the NMSSM that we will exploit in subsequent examples, since it allows for a significant increase in the cross section. The neutralinos in these regions have $m_{\tilde{\chi}_{1}^{0}} \gtrsim 70 \mathrm{GeV}$ and have a mixed singlino-Higgsino

\footnotetext{
${ }^{6}$ Since the exclusion area due to ZEPLIN I is similar to EDELWEISS we have not depicted it here, nor in any subsequent Figure.
} 

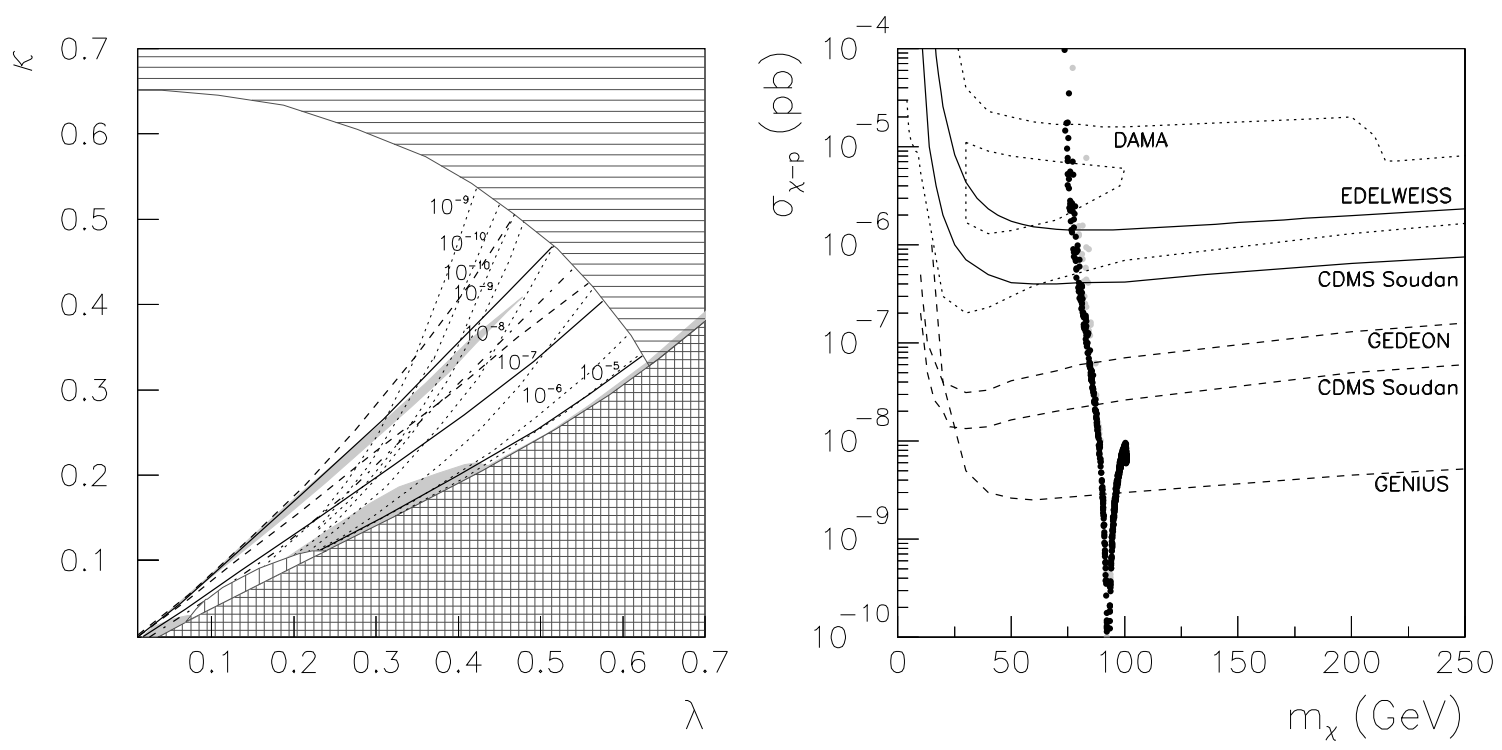

Figure 4: $(\lambda, \kappa)$ parameter space with the corresponding constraints and neutralino-nucleon cross section as a function of the lightest neutralino mass for the case $A_{\lambda}=200 \mathrm{GeV}$, $A_{\kappa}=-200 \mathrm{GeV}, \mu=110 \mathrm{GeV}$ and $\tan \beta=3$. In the $(\lambda, \kappa)$ plane the mass and composition of the lightest scalar Higgs, the composition of the lightest neutralino (only the line with $N_{15}^{2}=0.1$ ), and the predictions for $\sigma_{\tilde{\chi}_{1}^{0}-p}$ are represented with the same line conventions as in Fig. 2, and the new ruled area (vertical lines) is excluded due to the occurrence of unphysical minima. The colour convention for the plot $\sigma_{\tilde{\chi}_{1}^{0}-p}$ versus $m_{\tilde{\chi}_{1}^{0}}$ is as in Fig. 3.

composition $\left(N_{15}^{2} \lesssim 0.3\right.$ and $N_{13}^{2}+N_{14}^{2} \gtrsim 0.7$ in the region with larger cross section).

Notice that $\sigma_{\tilde{\chi}_{1}^{0}-p}$ displays an important suppression around $m_{\tilde{\chi}_{1}^{0}} \approx 90 \mathrm{GeV}$. This is due to the cancellation of the contribution of the cross section coming from neutralinoneutralino-Higgs interaction due to the occurrence of terms with different signs. This type of accidental cancellations is analogous to those appearing in MSSM analyses for $\mu<0$ [38].

These results are also sensitive to variations in the rest of the input parameters $\left(A_{\kappa}\right.$, $\tan \beta, \mu$, and $A_{\lambda}$ ). For instance, increasing $\left|A_{\kappa}\right|$ (i.e., making it more negative) leads to a further decrease in $\mathcal{M}_{S, 33}^{2}$ in the CP-even Higgs mass matrix, and therefore lighter Higgses can be obtained with a larger singlet composition. Although this translates into an enlargement of the regions where one has a tachyonic scalar Higgs, one may nevertheless find a larger $\sigma_{\tilde{\chi}_{1}^{0}-p}$ in the allowed areas. Choosing $\tan \beta=3, A_{\lambda}=200 \mathrm{GeV}$ and 
$\mu=110 \mathrm{GeV}$, but with $A_{\kappa}=-200 \mathrm{GeV}$, one can obtain $\sigma_{\tilde{\chi}_{1}^{0}-p} \gtrsim 10^{-4} \mathrm{pb}$ (points which in fact are already excluded by direct dark matter searches). The corresponding $(\lambda, \kappa)$ parameter space, as well as $\sigma_{\tilde{\chi}_{1}^{0}-p}$ versus the neutralino mass, are represented in Fig 4. Remarkably, very light Higgses are allowed in this case $\left(m_{h_{1}^{0}} \gtrsim 20 \mathrm{GeV}\right)$ due to their significant singlet character $\left(0.9 \lesssim S_{13}^{2} \lesssim 0.95\right)$. Once again, the lightest neutralino exhibits a large singlino-Higgsino composition $\left(N_{15}^{2} \lesssim 0.3\right.$ and $\left.N_{13}^{2}+N_{14}^{2} \gtrsim 0.7\right)$. For these reasons, one hardly finds experimentally excluded regions: only narrow stripes, mostly due to direct production of $\tilde{\chi}^{0}$ and $h^{0} \rightarrow b \bar{b}$. Also, for small values of $\lambda$ and $\kappa$, a very thin region excluded by the existence of false minima (see Section 2.5) appears. Conversely, decreasing $\left|A_{\kappa}\right|$ helps reducing tachyonic regions. In the particular case where $A_{\kappa}=0$, no tachyons emerge from the CP-even sector. The implications of this variation in the value of $\sigma_{\tilde{\chi}_{1}^{0}-p}$ are minimal.

Changing $\tan \beta$ has an important impact in the analysis, mainly due to the effect on the Higgs sector. The tachyonic regions become larger as $\tan \beta$ increases (extending towards higher values of $\lambda$ and $\kappa$ ). As a consequence, the neutralino is never a pure singlino and its mass increases due to the larger mixing with Higgsinos. For this reason the exclusion due to direct neutralino production becomes larger. In the end, not only the allowed region is reduced, but also the predictions for $\sigma_{\tilde{\chi}_{1}^{0}-p}$ are smaller. Also, for very small values of $\tan \beta$ very light Higgsino-like neutralinos can be found in large regions of the parameter space. The experimental constraints are, nevertheless, more important and only small areas survive. Fig. 5 illustrates these properties. 


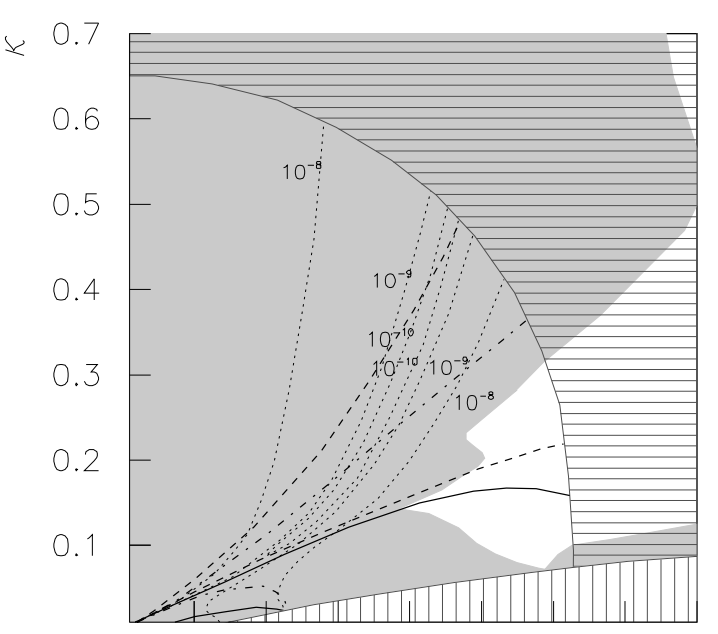

$\begin{array}{llllllll}0.1 & 0.2 & 0.3 & 0.4 & 0.5 & 0.6 & 0.7 & 0.8\end{array}$

$\lambda$

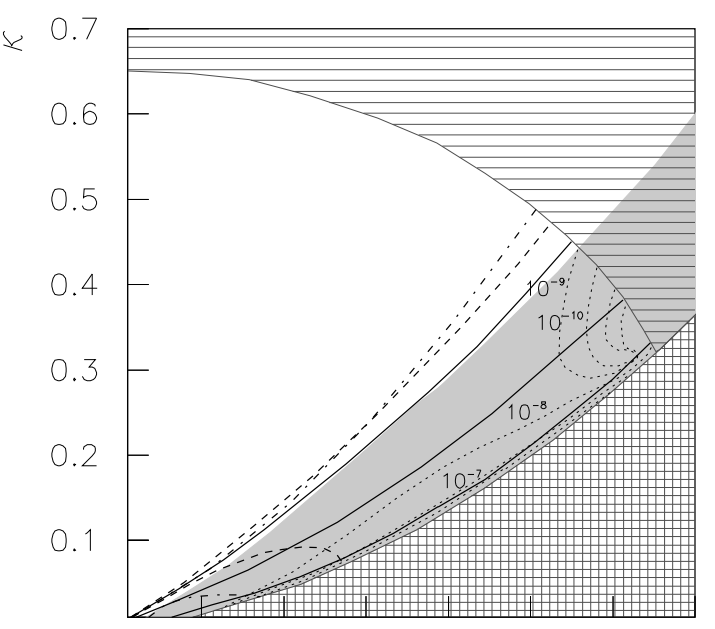

$\begin{array}{lllllll}0.1 & 0.2 & 0.3 & 0.4 & 0.5 & 0.6 & 0.7\end{array}$
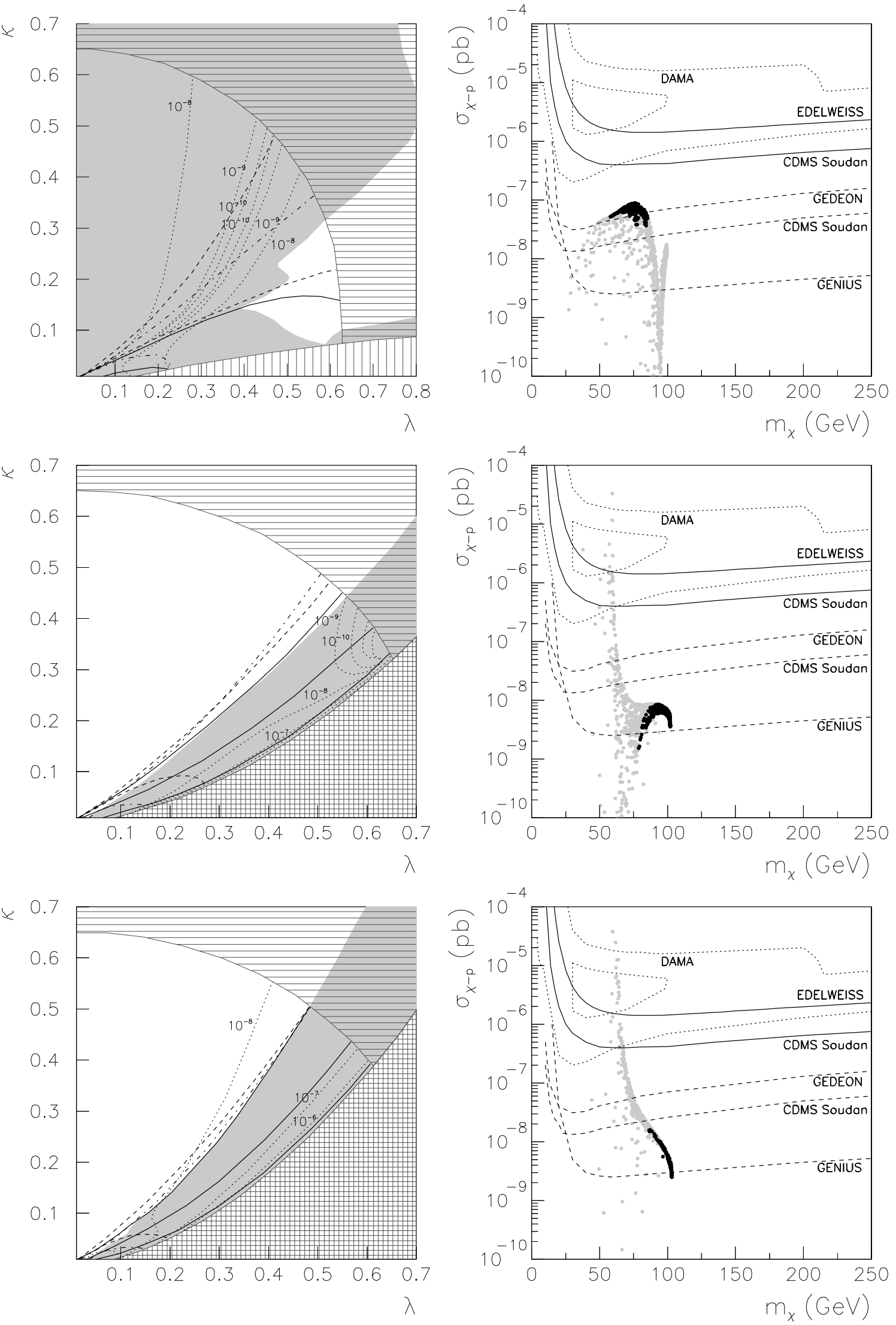

Figure 5: The same as in Fig. 4 but for the cases $A_{\lambda}=200 \mathrm{GeV}, A_{\kappa}=-50 \mathrm{GeV}, \mu=110$ $\mathrm{GeV}$, and $\tan \beta=2,5,10$, from top to bottom. In the case with $\tan \beta=2$, only the lines with $m_{h_{1}^{0}}=75,25 \mathrm{GeV}$ are represented, since $m_{h_{1}^{0}} \lesssim 110 \mathrm{GeV}$. 

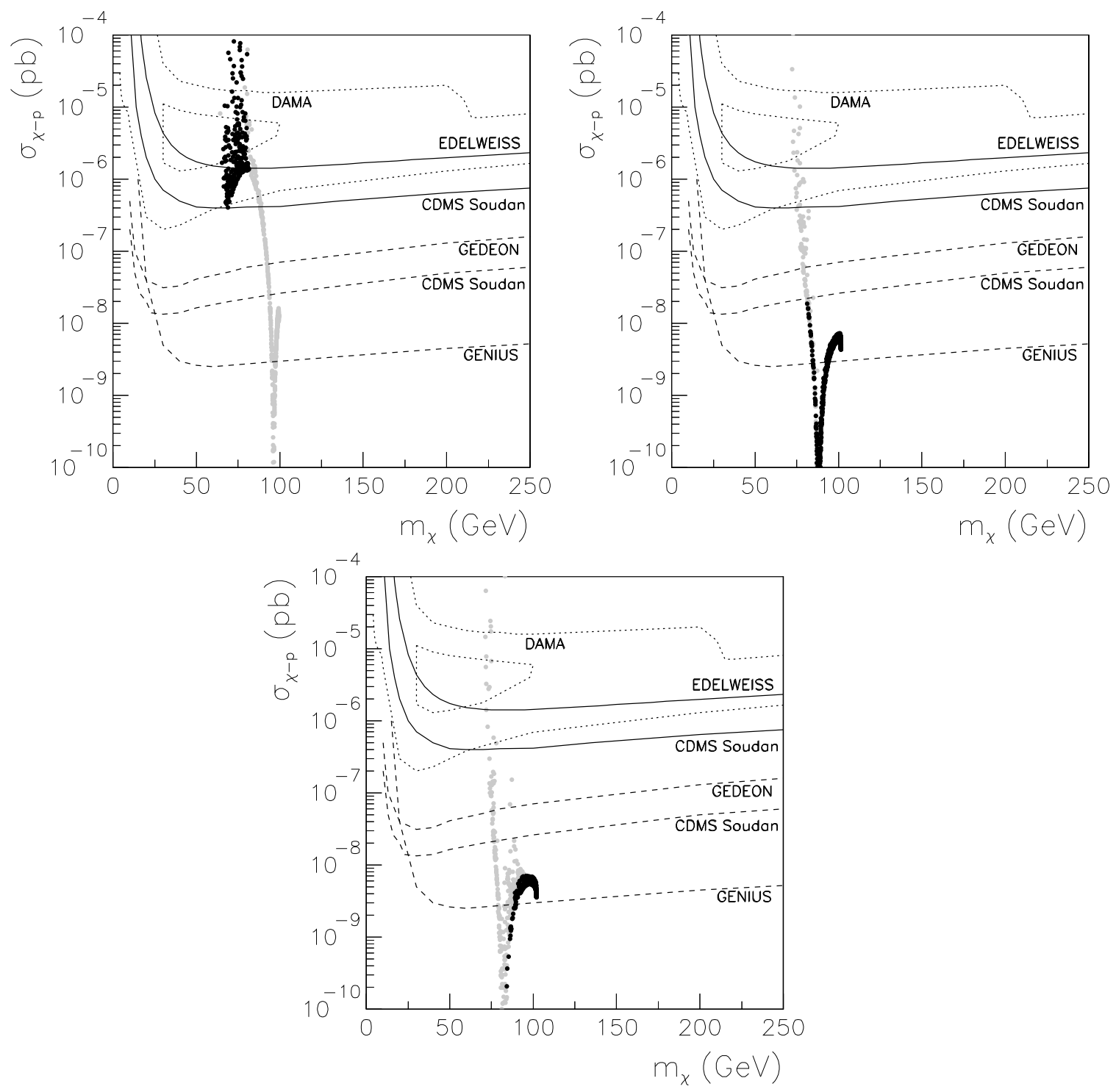

Figure 6: The same as in Fig. 3a but for the cases $A_{\lambda}=200 \mathrm{GeV}, A_{\kappa}=-200 \mathrm{GeV}$, $\mu=110 \mathrm{GeV}$, and $\tan \beta=2,4,5$, from left to right and top to bottom.

Similar examples, but for $A_{\kappa}=-200 \mathrm{GeV}$ can be found in Fig. 6 , where the predictions for $\sigma_{\tilde{\chi}_{1}^{0}-p}$ are depicted as a function of the neutralino mass for $\tan \beta=2,4,5$. As already mentioned, small values of $\tan \beta$ favour lighter neutralinos with larger detection cross section.

Heavier neutralinos with a larger singlino composition can be obtained if the value of $\mu$ is increased. For this reason, the regions where direct neutralino production is not 

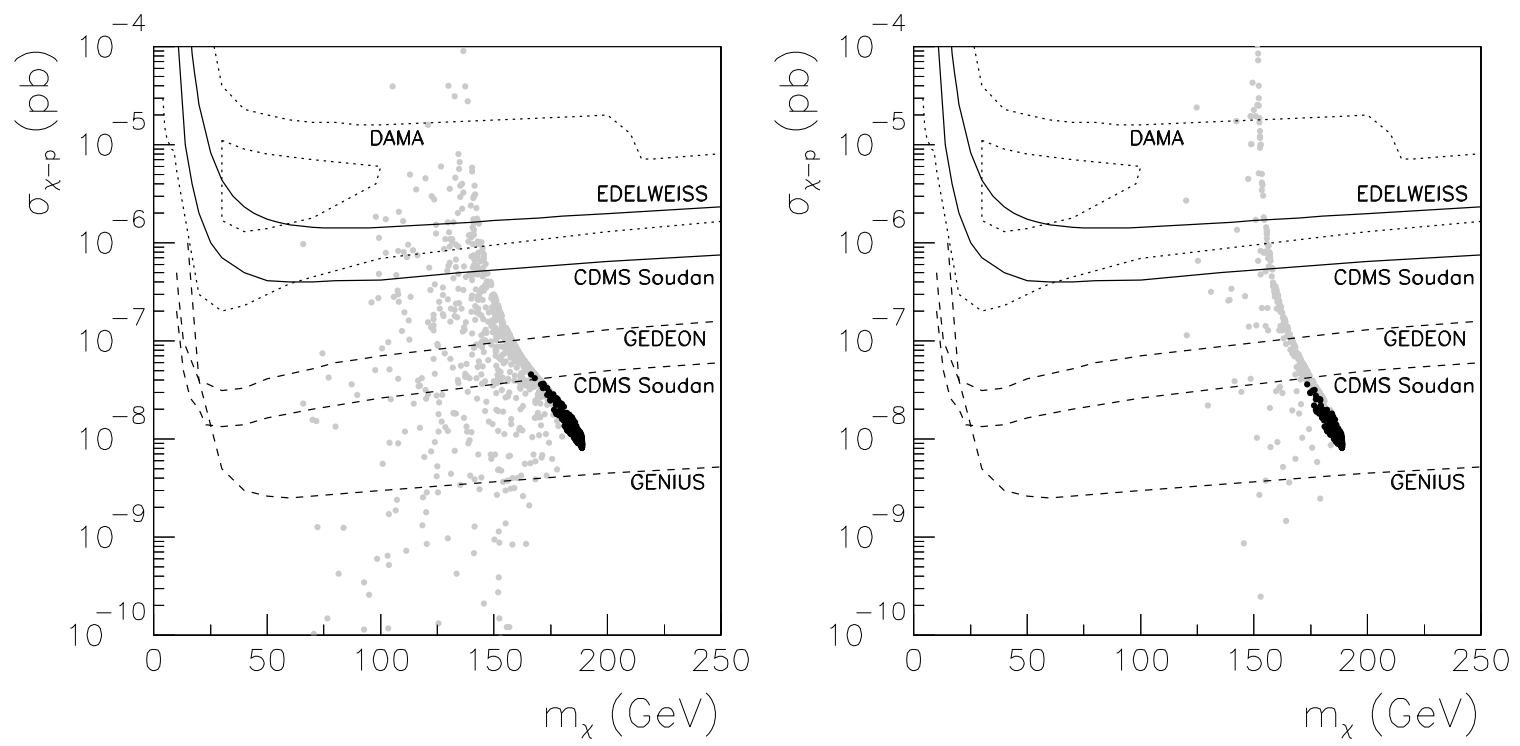

Figure 7: The same as in Fig. 3a but for the cases $A_{\lambda}=200 \mathrm{GeV}, \tan \beta=3, \mu=200 \mathrm{GeV}$, and $A_{\kappa}=-50,-200 \mathrm{GeV}$, from left to right.

in agreement with experimental bounds become much narrower. The mass of scalar Higgses also grows in this case, as well as their doublet character. Constraints on the Higgs sector are still strong enough to forbid those points where the neutralino is mostly singlino, and in the end $\tilde{\chi}_{1}^{0}$ preserves its mixed singlino-Higgsino character. In the remaining allowed area the predictions for $\sigma_{\tilde{\chi}_{1}^{0}-p}$ can vary, being typically smaller than in cases with low $\mu$. This is shown in Fig. 7 for two examples with $\mu=200 \mathrm{GeV}$, $A_{\lambda}=200 \mathrm{GeV}, \tan \beta=3$, and $A_{\kappa}=-50,-200 \mathrm{GeV}$. In particular, in the case with $A_{\kappa}=-200 \mathrm{GeV}$, the detection cross section is much smaller than in the analogous example with $\mu=110 \mathrm{GeV}$ presented in Fig. 4 .

Finally, variations in the value of $A_{\lambda}$ also influence the theoretical predictions on $\sigma_{\tilde{\chi}_{1}^{0}-p}$. There is a range of $A_{\lambda}$ for which the eigenvalues of the CP-even Higgs mass matrix are positive. However, for smaller or larger $A_{\lambda}$, off-diagonal terms may become large enough to ease the appearance of tachyons in the large $\lambda$ regime. For instance, in Fig. 8 we have represented the $(\lambda, \kappa)$ plane and the corresponding predictions for $\sigma_{\tilde{\chi}_{1}^{0}-p}$ in two cases with $A_{\kappa}=-50 \mathrm{GeV}, \mu=110 \mathrm{GeV}, \tan \beta=3$, and $A_{\lambda}=50,450 \mathrm{GeV}$. We find that, in agreement with the discussion above, the tachyonic regions are larger than those for $A_{\lambda}=200 \mathrm{GeV}$ in both cases. Also, the areas excluded by experimental constraints associated to IHDM and DHDM are more extensive, and in the case of $A_{\lambda}=450 \mathrm{GeV}$ they forbid most of the parameter space. The neutralino is mostly 

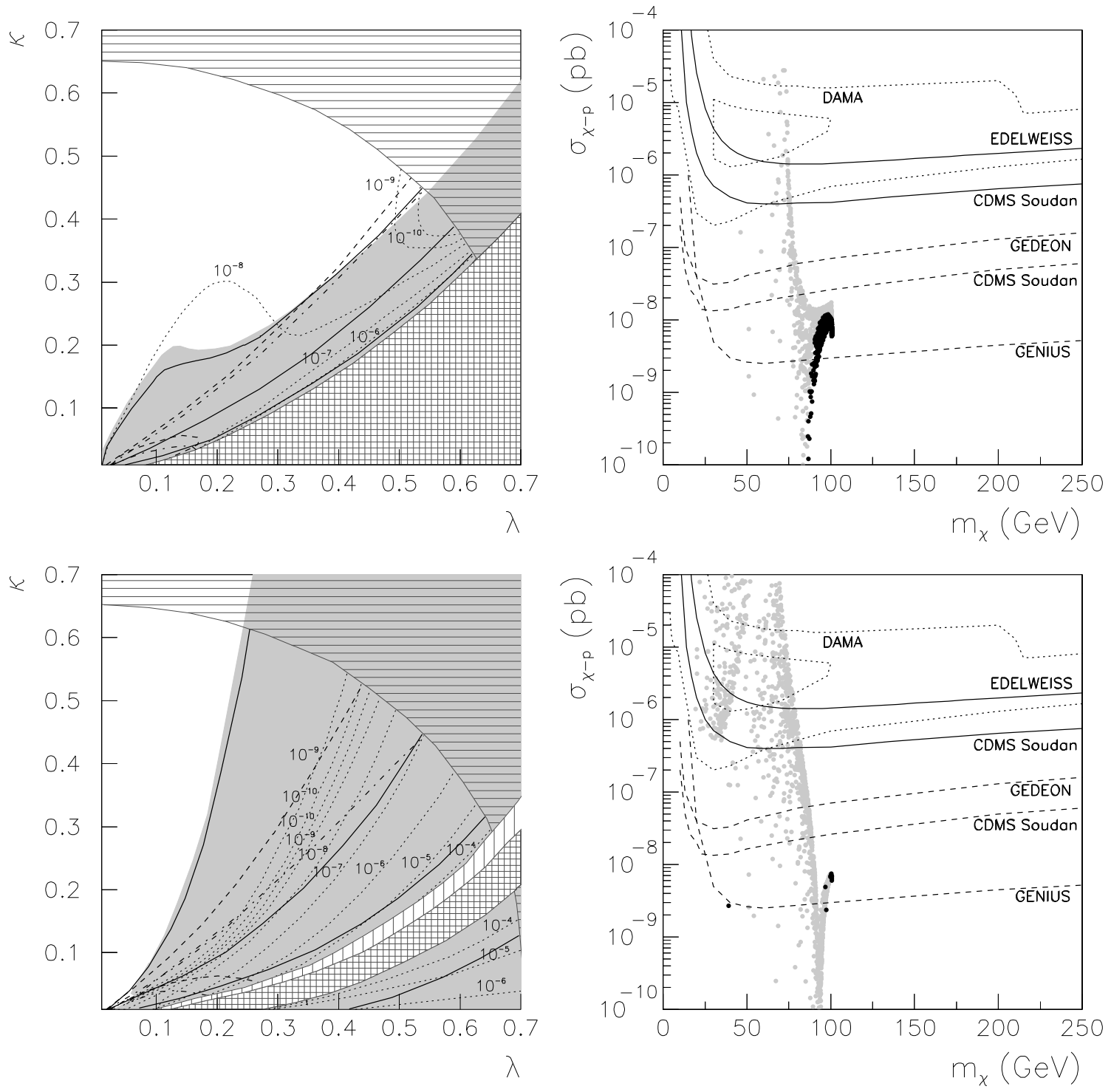

Figure 8: The same as in Fig. 4 but for the cases $A_{\kappa}=-50 \mathrm{GeV}, \mu=110 \mathrm{GeV}, \tan \beta=3$, and $A_{\lambda}=50,450 \mathrm{GeV}$, from top to bottom.

Higgsino in the remaining allowed points, with $N_{15}^{2} \lesssim 0.1(0.2)$ and $N_{13}^{2}+N_{14}^{2} \gtrsim 0.9(0.8)$ for $A_{\lambda}=50(450) \mathrm{GeV}$, and there is a slight decrease in the predictions for $\sigma_{\tilde{\chi}_{1}^{0}-p}$.

The range of values of $A_{\lambda}$ for which the allowed area is more extensive is very dependent on the rest of the inputs. In particular, since large $\tan \beta$ and $\left|A_{\kappa}\right|$ increase the diagonal term, $\mathcal{M}_{P, 22}^{2}$, in the CP-odd Higgs mass matrix, larger values of $A_{\lambda}$ can be taken before $\mathcal{M}_{P, 12}^{2}$ gets too big. For example, in the case with $A_{\kappa}=-200 \mathrm{GeV}$ one 

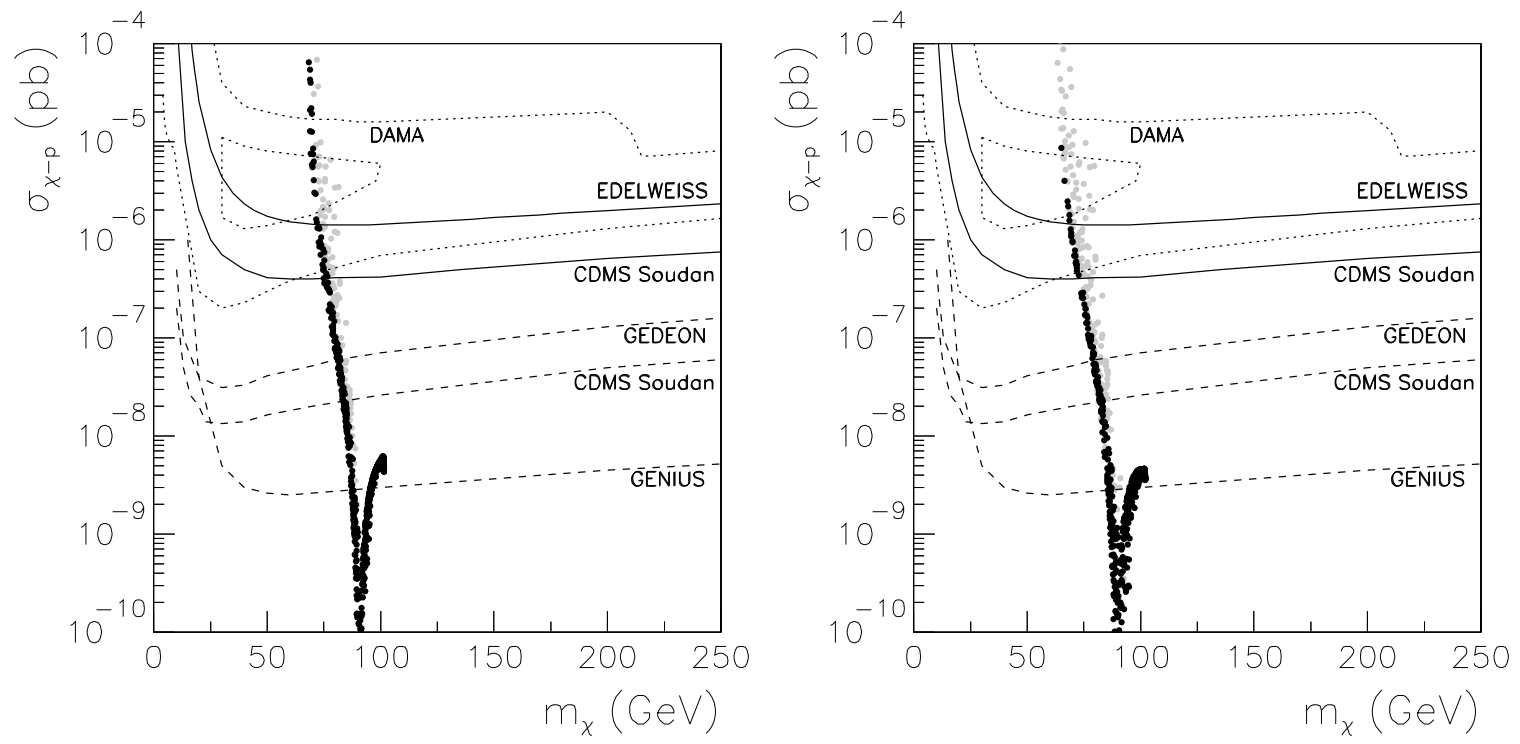

Figure 9: The same as in Fig. 3a but for the cases $A_{\kappa}=-200 \mathrm{GeV}, \mu=110 \mathrm{GeV}$, $\tan \beta=4$ and $A_{\lambda}=300 \mathrm{GeV}$, on the left, and $A_{\kappa}=-200 \mathrm{GeV}, \mu=110 \mathrm{GeV}, \tan \beta=5$ and $A_{\lambda}=450 \mathrm{GeV}$ on the right.

can still obtain large accepted regions for $A_{\lambda}=300-450 \mathrm{GeV}$ and $\tan \beta=4-5$, as evidenced in Fig. 9, where points entering the sensitivities of the present dark matter detectors are obtained with $m_{\tilde{\chi}_{1}^{0}} \lesssim 75 \mathrm{GeV}$.

To complete the analysis of the cases with $\mu A_{\lambda}>0$ and $\mu A_{\kappa}<0$, we must address the possibility of having $\mu, A_{\lambda},-A_{\kappa}<0$. Note from (2.5) that the tree-level potential, $V_{\text {neutral }}^{\text {Higg }}$, and therefore the Higgs mass matrices, are invariant under the exchange of the signs of $\mu, A_{\lambda}$ and $A_{\kappa}$, provided that the signs of $\mu A_{\lambda}$ and $\mu A_{\kappa}$ do not change. This implies that the above analysis regarding the Higgs sector is identical in this case. Differences arise, however, in the neutralino sector since the signs of $M_{1,2}$ were not altered. Therefore, the neutralino mass spectrum differs, as well as the lightest neutralino composition. Also the experimental constraints exhibit a slight variation. This case presents the same qualitative behaviour as the one formerly discussed in what the minimization of the Higgs potential is concerned. Nevertheless, differences arise regarding the theoretical predictions for $\sigma_{\tilde{\chi}_{1}^{0}-p}$ due to the experimental constraints and the different position of the accidental suppressions in the Higgs-exchange diagrams. These differences can be sizable for large $\tan \beta$. For instance, we have represented in Fig. 10 two examples with $A_{\lambda}=-200 \mathrm{GeV}, \mu=-110 \mathrm{GeV}, \tan \beta=3$, and $A_{\kappa}=50,200 \mathrm{GeV}$, where the suppression in $\sigma_{\tilde{\chi}_{1}^{0}-p}$ is found to occur for $m_{\tilde{\chi}_{1}^{0}} \approx 110 \mathrm{GeV}$. 

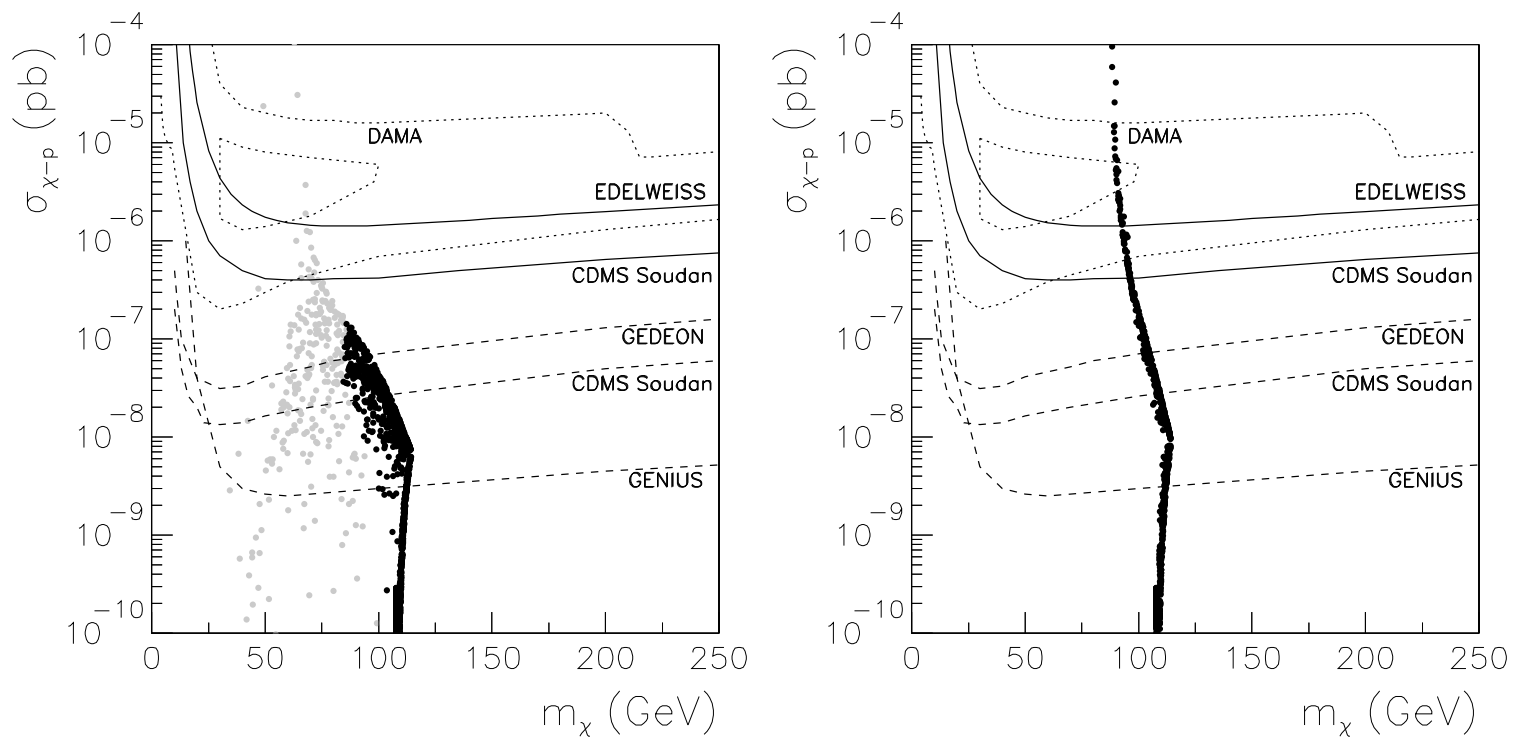

Figure 10: The same as in Fig. 3a but for the cases $A_{\lambda}=-200 \mathrm{GeV}, \mu=-110 \mathrm{GeV}$, $\tan \beta=3$, and $A_{\kappa}=50,200 \mathrm{GeV}$, from left to right.

To sum up, we have found that large values of $\sigma_{\tilde{\chi}_{1}^{0}-p}$, even within the reach of dark matter detectors, can be obtained in the scenarios analysed in this Subsection. The NMSSM nature is evidenced in these examples by the compositions of the lightest neutralino (which is a singlino-Higgsino mixed state) and the scalar Higgs (which can be mostly singlet and as light as $m_{h_{1}^{0}} \gtrsim 20 \mathrm{GeV}$ ).

\section{$4.2 \mu A_{\kappa}<0$ and $\mu A_{\lambda}<0(\kappa>0)$}

This choice comprises the cases $\mu,-A_{\lambda},-A_{\kappa}>0$ and $\mu,-A_{\lambda},-A_{\kappa}<0$.

We first address the possibility $\mu,-A_{\lambda},-A_{\kappa}>0$. When compared with the cases discussed in the previous Subsection, the occurrence of tachyons in the Higgs sector gives rise to stronger constraints in this case, both in the CP-even and CP-odd Higgses.

For CP-even Higgses tachyons are now more likely to occur, due to the negative contributions in $\mathcal{M}_{S, 33}^{2}$, induced by the terms proportional to $\mu A_{\kappa}$ and $A_{\lambda} / \mu$. Similarly, $\mathcal{M}_{S, 11}^{2}$ receives a sizeable negative contribution from the term proportional to $\mu A_{\lambda}$ which is particularly dangerous for large values of $\tan \beta$. In the CP-odd sector, an analogous study of the mass matrix shows that tachyons are more restrictive for large values of $\lambda$ and small values of $\kappa$. Actually, from the naive requirement $\mathcal{M}_{P, 11}^{2} \geq 0$ the 
following constraint is obtained $\kappa \geq-\lambda A_{\lambda} / \mu$. In fact, this ensures the positiveness of the denominator in condition (ii) derived from the minimization of the Higgs potential in Section 2.2, and gives a qualitative idea on the dependence of the tachyonic region on the parameters $A_{\lambda}$ and $\mu$. When compared with the cases treated in the former Subsection, larger regions of the parameter space are now excluded. We found that tachyons in the CP-odd sector typically give rise to stronger constraints than those from CP-even sector, although the corresponding excluded regions practically coincide.

The experimental constraints from the neutralino sector are not very stringent in these examples, owing to the fact that the regions where the neutralino would have a small mass are typically excluded by the occurrence of tachyons.

As an example, the $(\lambda, \kappa)$ plane is represented in Fig. 11 for $\tan \beta=3$, $A_{\lambda}=-200 \mathrm{GeV}, A_{\kappa}=-50 \mathrm{GeV}$ and $\mu=110 \mathrm{GeV}$. In this case, and in contrast with what was displayed in Fig. 2, there exists a very large region where one cannot find minima of $V_{\text {neutral }}^{\text {Higgs }}$. In particular, $\lambda \gtrsim 0.25$ is now excluded for this reason. In the rest of the parameter space, experimental constraints become very important in those regions with small values of the $\mathrm{CP}$-even and $\mathrm{CP}$-odd masses. Although the most important exclusion is due to DHDM constraints $\left(h^{0} \rightarrow b \bar{b}\right.$ and $\left.h^{0} \rightarrow \tau^{+} \tau^{-}\right)$, some regions not fulfilling the bounds on APM $\left(h^{0} a^{0} \rightarrow 4 b\right.$ 's) also appear. It is worth emphasizing that in the remaining allowed regions the lightest Higgs is doublet-like $\left(S_{13}^{2} \lesssim 0.003\right)$ and its mass is never too small, $m_{h_{1}^{0}} \gtrsim 85 \mathrm{GeV}$.

Regarding the composition of the lightest neutralino, it turns out to be Higgsino-like in all the allowed parameter space $\left(N_{13}^{2}+N_{14}^{2} \gtrsim 0.98\right)$. As we already mentioned, those regions with small $\lambda$ and $\kappa$ that would lead to a singlino-like neutralino are excluded by the absence of physical minima in the potential. For this reason the mass of the neutralino is dictated by the value of the $\mu$ term and we found $m_{\tilde{\chi}_{1}^{0}} \approx \mu$ throughout the allowed parameter space.

This is shown in Fig. 11, which represents the corresponding values of the neutralinonucleon cross section as a function of the neutralino mass. The cross section ranges from $6 \times 10^{-9} \mathrm{pb} \lesssim \sigma_{\tilde{\chi}_{1}^{0}-p} \lesssim 7 \times 10^{-8} \mathrm{pb}$ in this case. Once more, although higher values could be obtained, these are typically excluded due to the constraints on the CP-even Higgses.

Let us now address the relevance of variations in $A_{\kappa}$ in the allowed regions of the parameter space and thus on the predictions for $\sigma_{\tilde{\chi}_{1}^{0}-p}$. It can be seen that the increase in $\left|A_{\kappa}\right|$ (i.e., making it more negative) translates into an almost negligible enlargement 

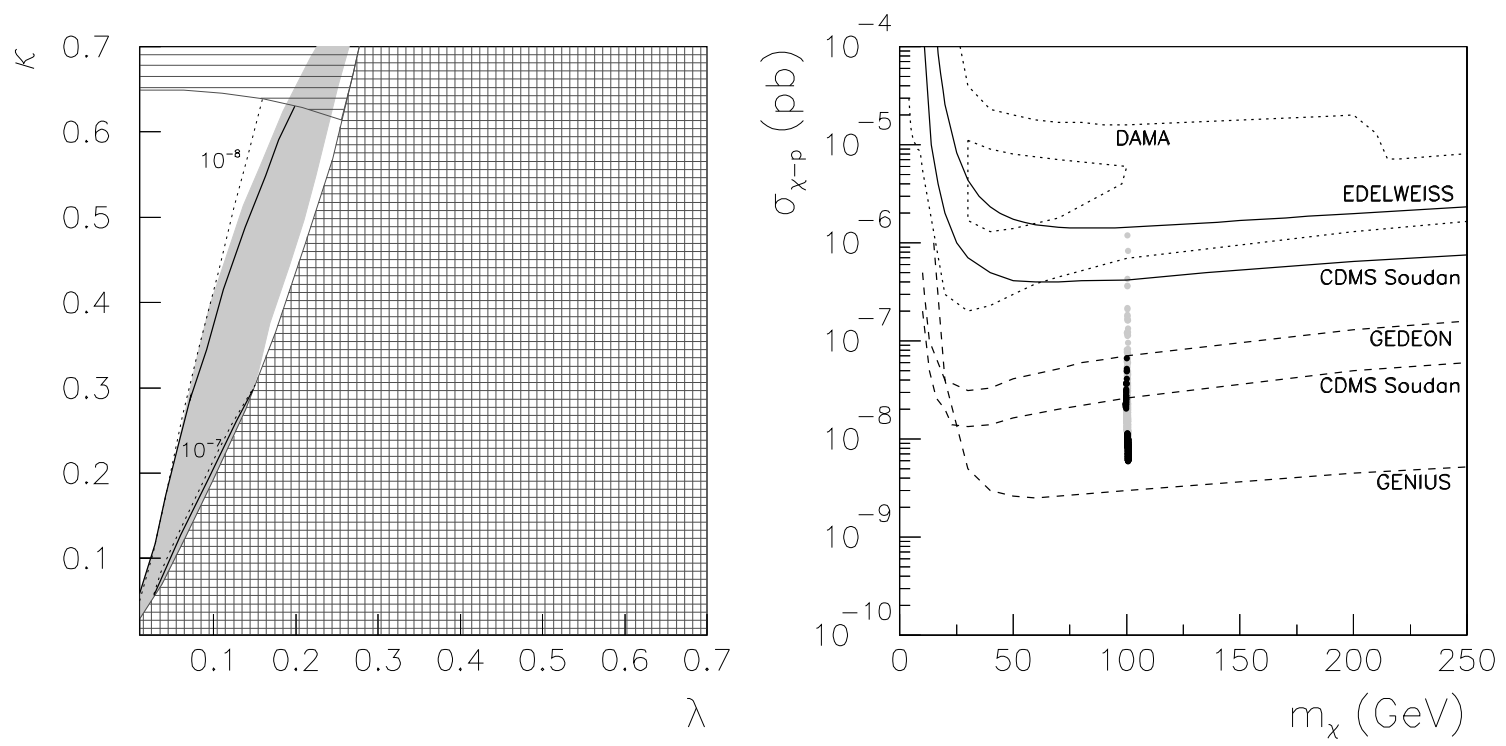

Figure 11: The same as in Fig. 4 but for $\tan \beta=3, A_{\lambda}=-200 \mathrm{GeV}, A_{\kappa}=-50 \mathrm{GeV}$ and $\mu=110 \mathrm{GeV}$. Only the lines with $m_{h_{1}^{0}}=114,75 \mathrm{GeV}$ are represented, and none of the lines showing the lightest scalar Higgs and neutralino composition is depicted, since $S_{13}^{2}<0.1$ and $N_{15}^{2}<0.1$ in all the plane.

in the allowed area, while the experimental constraints on CP-even Higgses become more restrictive. On the other hand, a decrease in the value of $\left|A_{\kappa}\right|$ leads to a lighter CP-odd Higgs and the tachyonic region increases, as can be easily understood from the mass matrix (2.10). For instance, in the particular case of $A_{\kappa}=0$, and unless $\left|A_{\lambda}\right|$ is also very small, the entire parameter space can be excluded.

Regarding changes in $A_{\lambda}$ and $\mu$, these clearly affect the regions excluded by tachyons. Large values of $\mu$ and small $\left|A_{\lambda}\right|$ allow an increase in the accepted regions, in agreement with the condition on $\kappa$ derived above, $\kappa \geq-\lambda A_{\lambda} / \mu$. Also, note that, since the masses of the Higgses increase, the associated experimental constraints become less restrictive and the allowed area is larger. Nevertheless, the region where the neutralino would have an important singlino composition is still typically excluded, and therefore in the allowed region $\tilde{\chi}_{1}^{0}$ is still Higgsino-like, with $m_{\tilde{\chi}_{1}^{0}} \approx \mu$. Despite the increase of $m_{\tilde{\chi}_{1}^{0}}$, the predictions for the cross section are essentially unaltered. An example with $\tan \beta=3$, $A_{\lambda}=-200 \mathrm{GeV}, A_{\kappa}=-50 \mathrm{GeV}$ and $\mu=200 \mathrm{GeV}$ is represented in Fig. 12, displaying both the $(\lambda, \kappa)$ plane and the neutralino-nucleon cross section versus the neutralino mass. We find $\sigma_{\tilde{\chi}_{1}^{0}-p} \lesssim 5 \times 10^{-8} \mathrm{pb}$, similar to what was found in Fig. 11, but now with $m_{\tilde{\chi}_{1}^{0}} \approx 190 \mathrm{GeV}$. The singlino component of $\tilde{\chi}_{1}^{0}$ is negligible $\left(N_{15}^{2} \lesssim 0.006\right)$ and 

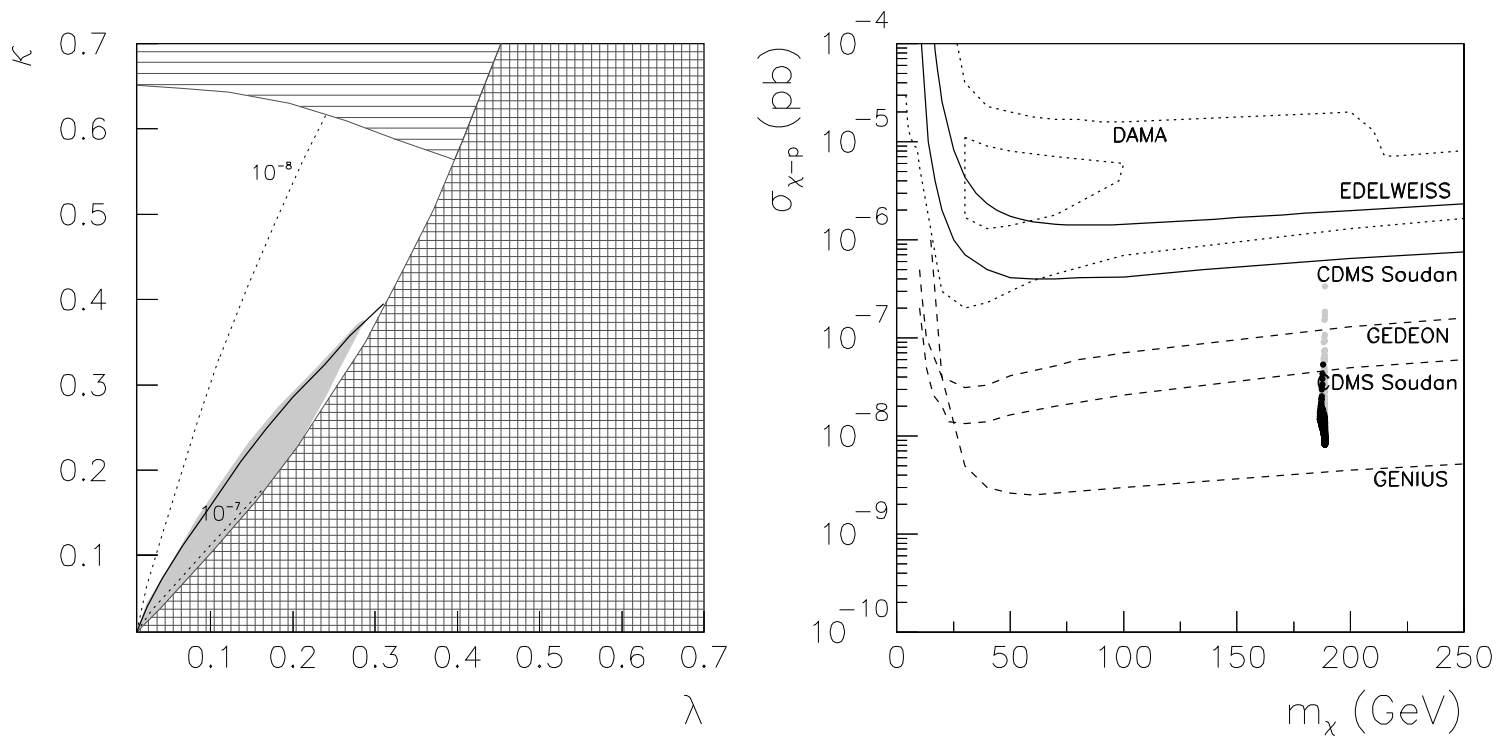

Figure 12: The same as in Fig. 4 but for $\tan \beta=3, A_{\lambda}=-200 \mathrm{GeV}, A_{\kappa}=-50 \mathrm{GeV}$ and $\mu=200 \mathrm{GeV}$. Only the line with $m_{h_{1}^{0}}=114 \mathrm{GeV}$ is represented, and none of the lines showing the lightest scalar Higgs and neutralino composition is depicted, since $S_{13}^{2}<0.1$ and $N_{15}^{2}<0.1$ in all the plane.

the scalar Higgs is doublet-like $\left(S_{13}^{2} \lesssim 0.001\right)$.

Finally, regarding variations in the value of $\tan \beta$, these have little effect on the shape of the tachyonic region, whereas experimental constraints are more sensitive to them. As in the former scenario, for low values of $\tan \beta$ light scalar Higgses are obtained. Since these are predominantly doublet-like, experimental constraints (especially those associated with DHDM, namely, $h^{0} \rightarrow b \bar{b}$ and $h^{0} \rightarrow \tau^{+} \tau^{-}$) become very important and forbid, for instance, the whole parameter space in the case $\tan \beta=2$. On the other hand, larger values of $\tan \beta$ are welcome in order to increase the value of $m_{h_{1}^{0}}$, obtaining also a moderate enhancement of the cross section. In order to illustrate this discussion, we represent in Fig. 13 two cases with $\tan \beta=2,5$, for $A_{\lambda}=-200 \mathrm{GeV}, A_{\kappa}=-50 \mathrm{GeV}$ and $\mu=110 \mathrm{GeV}$. We find that the cross section can reach $\sigma_{\tilde{\chi}_{1}^{0}-p} \approx 10^{-7} \mathrm{pb}$ in the case where $\tan \beta=5$. None of these examples displays any qualitative change regarding the neutralino and Higgs compositions.

To complete the analysis of the cases with $\mu A_{\lambda}<0$ we still have to consider the case $\mu,-A_{\lambda},-A_{\kappa}<0$. As we explained in the former Subsection, the analysis of the Higgs sector will be analogous to that of the case we have just studied. Despite the 

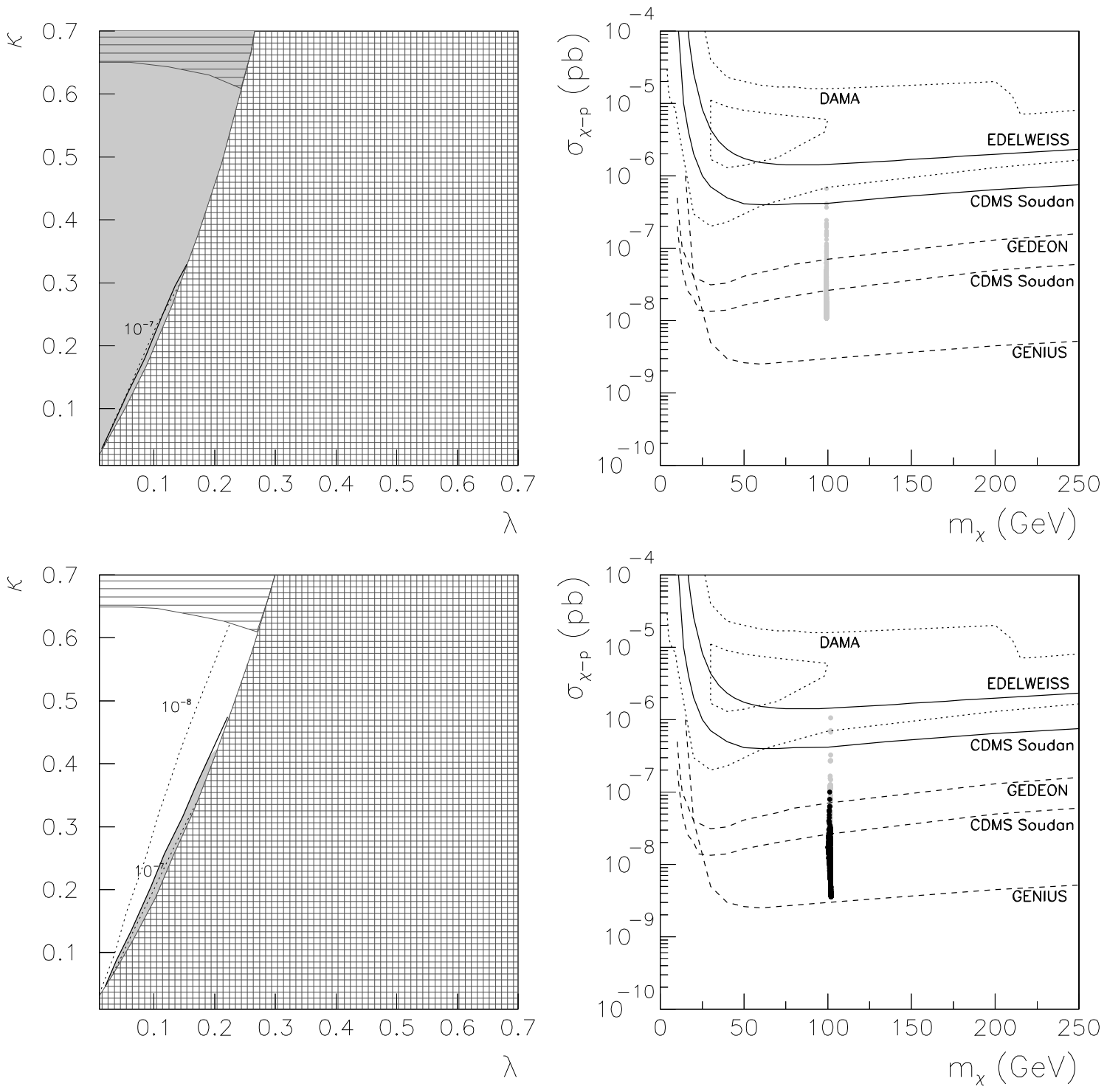

Figure 13: The same as in Fig. 4 but for the cases $A_{\lambda}=-200 \mathrm{GeV}, A_{\kappa}=-50 \mathrm{GeV}$, $\mu=110 \mathrm{GeV}$, and $\tan \beta=2,5$, from top to bottom. In the left frames, only the line with $m_{h_{1}^{0}}=114 \mathrm{GeV}$ is represented, and none of the lines showing the lightest scalar Higgs and neutralino composition is depicted, since $S_{13}^{2}<0.1$ and $N_{15}^{2}<0.1$ in all the plane. 
differences in the neutralino sector, its mass, composition and detection cross section will also be qualitatively equal to those previously discussed.

Summarizing, all the cases we have analysed in this Subsection present as common features the appearance of Higgsino-like neutralinos with a detection cross section which can be as large as $\sigma_{\tilde{\chi}_{1}^{0}-p} \lesssim 10^{-7} \mathrm{pb}$, and doublet-like Higgses.

\section{$4.3 \mu A_{\kappa}>0$ and $\mu A_{\lambda}>0(\kappa>0)$}

We consider now those cases where $\mu A_{\kappa}>0$, and $\mu A_{\lambda}>0$, conditions which are fulfilled in the cases $\mu, A_{\lambda}, A_{\kappa}>0$ and $\mu, A_{\lambda}, A_{\kappa}<0$.

We will begin with all $\mu, A_{\lambda}$, and $A_{\kappa}$ positive. As in the previous cases, a simple analysis of the tree-level Higgs mass matrices gives a qualitative understanding on the nature and extension of the tachyonic regions in the parameter space.

In this particular case tachyons in the CP-odd sector arise through the negative contribution $-\frac{3 \kappa \mu}{\lambda} A_{\kappa}$ in $\mathcal{M}_{P, 22}^{2}$. Since this is mainly compensated by the positive term $\frac{\lambda^{2} v^{2}}{\mu} A_{\lambda} \sin 2 \beta$, the tachyonic region occurs for small values of $\lambda$. The excluded region is obviously more important for small values of $A_{\lambda}$ and large $\mu, A_{\kappa}$, and $\tan \beta$. The occurrence of tachyons in the CP-even Higgses is analogous to the case $\mu A_{\lambda}>0$, $\mu A_{\kappa}<0$ discussed in Subsection 4.1, due to the increase of the off-diagonal terms in the mass matrix. As in that case, tachyons appear for large values of $\lambda$ and small $\kappa$ and become more stringent as $\tan \beta$ grows.

Experimental constraints play also a very relevant role in this case. Close to the tachyonic regions the experimental bounds on the Higgs sector are very severe. In particular IHDM, DHDM ( $h^{0} \rightarrow b \bar{b}, h^{0} \rightarrow 2$ jets) are responsible for the most important exclusions, although APM (mainly $h^{0} a^{0} \rightarrow 4 b$ 's) may also be violated. Finally, excluding those regions where the direct neutralino production is in disagreement with the experimental bounds leads to important constraints in the region with light $\tilde{\chi}_{1}^{0}$.

An example with $A_{\lambda}=200 \mathrm{GeV}, \mu=110 \mathrm{GeV}, A_{\kappa}=50 \mathrm{GeV}$ and $\tan \beta=3$ is represented in Fig. 14, depicting the constraints on the $(\lambda, \kappa)$ plane and the corresponding predictions for $\sigma_{\tilde{\chi}_{1}^{0}-p}$ versus the neutralino mass. In the small experimentally allowed region the lightest neutralino is a mixed singlino-Higgsino state, with $N_{15}^{2} \lesssim 0.4$ and $N_{13}^{2}+N_{14}^{2} \gtrsim 0.6$, and the lightest scalar Higgs can have an important singlet component $\left(S_{13}^{2} \lesssim 0.8\right)$. The experimental constraints impose $m_{\tilde{\chi}_{1}^{0}} \gtrsim 70 \mathrm{GeV}$ and $m_{h_{1}^{0}} \gtrsim 85 \mathrm{GeV}$, which set a limit on the theoretical predictions for the neutralino-nucleon cross section 

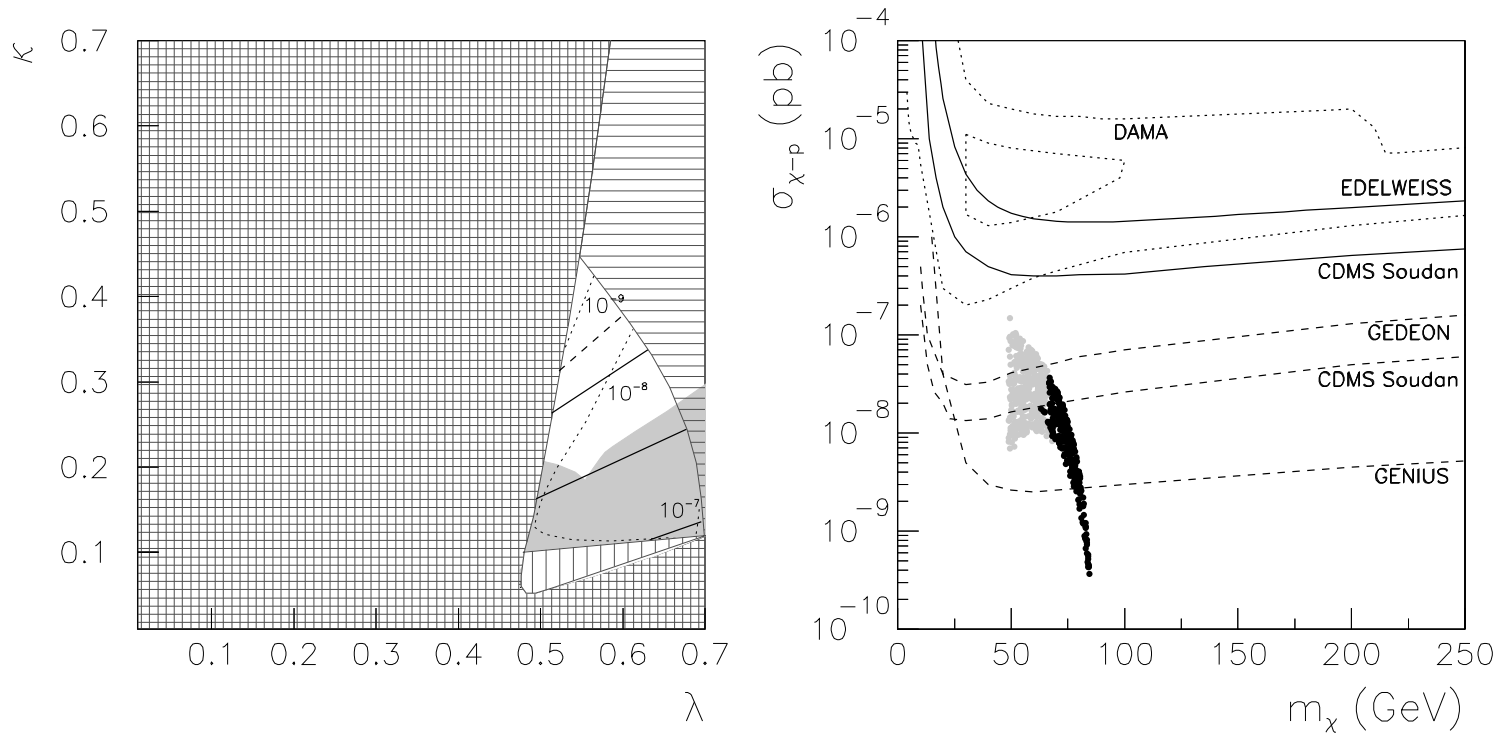

Figure 14: The same as in Fig. 4 but for $A_{\lambda}=200 \mathrm{GeV}, \mu=110 \mathrm{GeV}, A_{\kappa}=50 \mathrm{GeV}$, and $\tan \beta=3$. Only the line with $S_{13}^{2}=0.1$ is represented, and none of the lines showing the neutralino composition is depicted since $0.5>N_{15}^{2}>0.1$ in all the plane.

at $\sigma_{\tilde{\chi}_{1}^{0}-p} \lesssim 4 \times 10^{-8} \mathrm{pb}$.

Variations of $A_{\kappa}$ have an important impact on the allowed parameter space. As already commented, the region excluded due to tachyons in the CP-odd Higgs sector increases for larger values of $A_{\kappa}$. For instance, in the example with $A_{\lambda}=200 \mathrm{GeV}, \mu=$ $110 \mathrm{GeV}$, and $\tan \beta=3$, the allowed region completely disappears for $A_{\kappa} \gtrsim 110 \mathrm{GeV}$. On the other hand, decreasing the value of $A_{\kappa}$ the parameter space is enlarged. Recall that the minimal value $A_{\kappa}=0$ has already been analysed in Section 4.1 in the context of a scenario with $\mu, A_{\lambda},-A_{\kappa}>0$.

Decreasing the value of $A_{\lambda}$ also leads to an increase of regions with a tachyonic pseudoscalar. If $\mu=110 \mathrm{GeV}, A_{\kappa}=50$ and $\tan \beta=3$, the whole parameter space is excluded for $A_{\lambda} \lesssim 50 \mathrm{GeV}$. On the other hand, a moderate increase of $A_{\lambda}$ helps avoiding tachyons, especially in the CP-even sector. An example with $A_{\lambda}=300 \mathrm{GeV}$ can be found in Fig. 15, where the $(\lambda, \kappa)$ plane and the theoretical predictions for $\sigma_{\tilde{\chi}_{1}^{0}-p}$ are represented. Since in this case the experimental constraints from Higgs decays are less severe, we find that the regions with very light Higgs and $\tilde{\chi}_{1}^{0}$ are now experimentally viable. In particular, neutralinos with an important singlino composition, $N_{15}^{2} \lesssim 0.45$, can be obtained with $m_{\tilde{\chi}_{1}^{0}} \gtrsim 45 \mathrm{GeV}$, whereas the lightest Higgses $\left(m_{h_{1}^{0}} \approx 65-90 \mathrm{GeV}\right)$ 

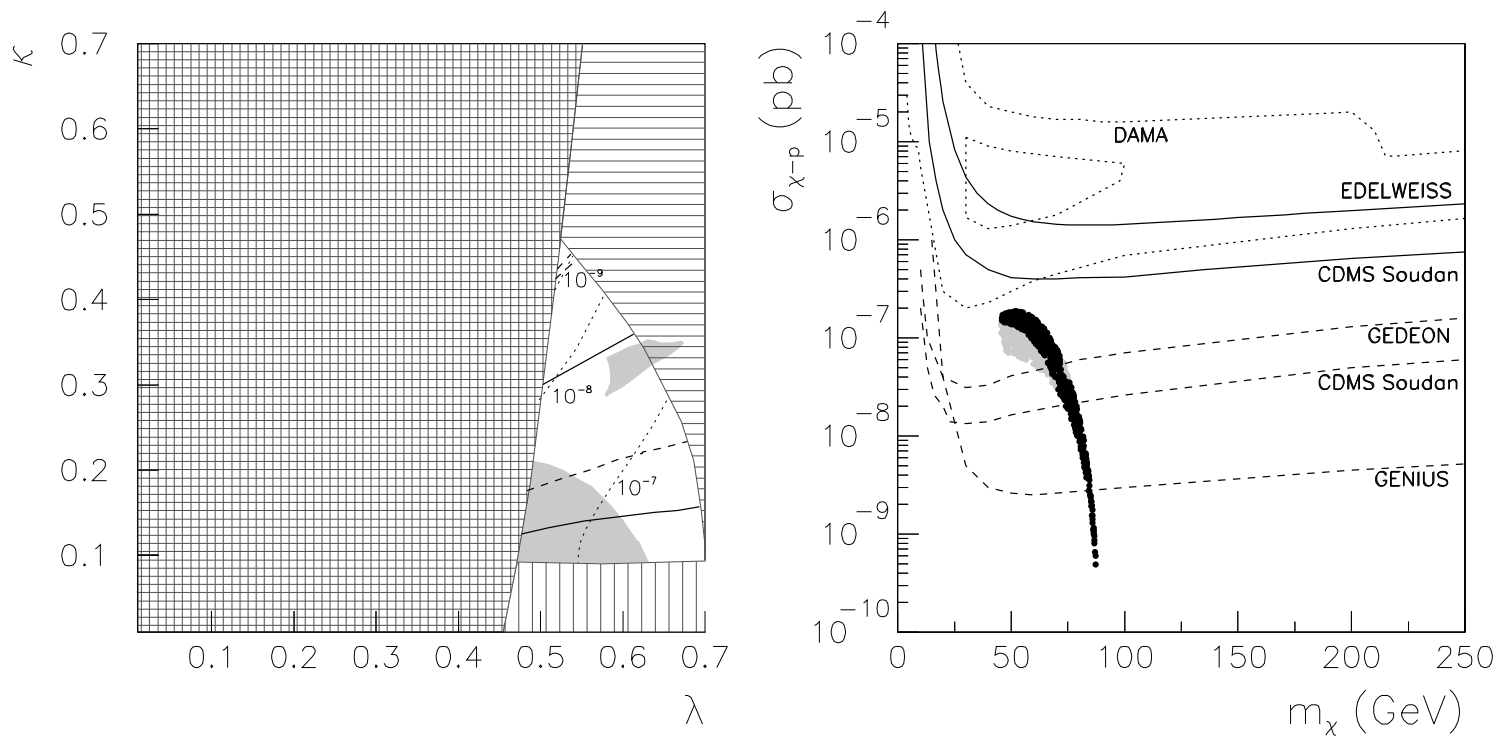

Figure 15: The same as in Fig. 4 but for $A_{\lambda}=300 \mathrm{GeV}, \mu=110 \mathrm{GeV}, A_{\kappa}=50 \mathrm{GeV}$, and $\tan \beta=3$. Only the lines with $m_{h_{1}^{0}}=114,75 \mathrm{GeV}$ are represented. Regarding the neutralino composition, only the line with $N_{15}^{2}=0.1$ is shown in the upper corner of the allowed region, since in the rest of the parameter space $0.5>N_{15}^{2}>0.1$.

are all singlet-like. This in turn favours larger values of the cross section $\left(\sigma_{\tilde{\chi}_{1}^{0}-p} \lesssim 2 \times\right.$ $10^{-7} \mathrm{pb}$ ), and compatibility with present experiments is almost obtained. Should we further increase the value of $A_{\lambda}$, the experimental constraints associated with the scalar Higgs would become again more important.

In order to prevent the occurrence of tachyons in the CP-odd Higgses, the value of $\mu$ has to be small. For instance, taking $\mu=200 \mathrm{GeV}$ in the example with $A_{\lambda}=200 \mathrm{GeV}$, $A_{\kappa}=50 \mathrm{GeV}$ and $\tan \beta=3$, all the $(\lambda, \kappa)$ plane would be excluded.

Regarding the value of $\tan \beta$, as already mentioned, the larger it is, the more extensive the regions excluded by $m_{h_{1}^{0}}^{2}<0$ become. In Fig. 16 we represent two examples with $A_{\lambda}=200 \mathrm{GeV}, A_{\kappa}=50 \mathrm{GeV}, \mu=110 \mathrm{GeV}$ and $\tan \beta=2,5$. We find that low values of $\tan \beta$ still allow physical minima of the potential. Moreover, both the singlino component of $\tilde{\chi}_{1}^{0}$ and the singlet component of the scalar Higgs can be slightly enhanced. For example, for $\tan \beta=2$ light neutralinos $\left(\tilde{\chi}_{1}^{0} \gtrsim 60 \mathrm{GeV}\right)$ can be obtained with $N_{15}^{2} \lesssim 0.55$, while Higgses in the mass range $m_{h_{1}^{0}} \approx 75-100 \mathrm{GeV}$ are singlet-like. However, the predictions for the detection cross section suffer a moderate decrease and only $\sigma_{\tilde{\chi}_{1}^{0}-p} \lesssim 5 \times 10^{-8} \mathrm{pb}$ is obtained. On the other hand, for $\tan \beta \gtrsim 5$ the whole 

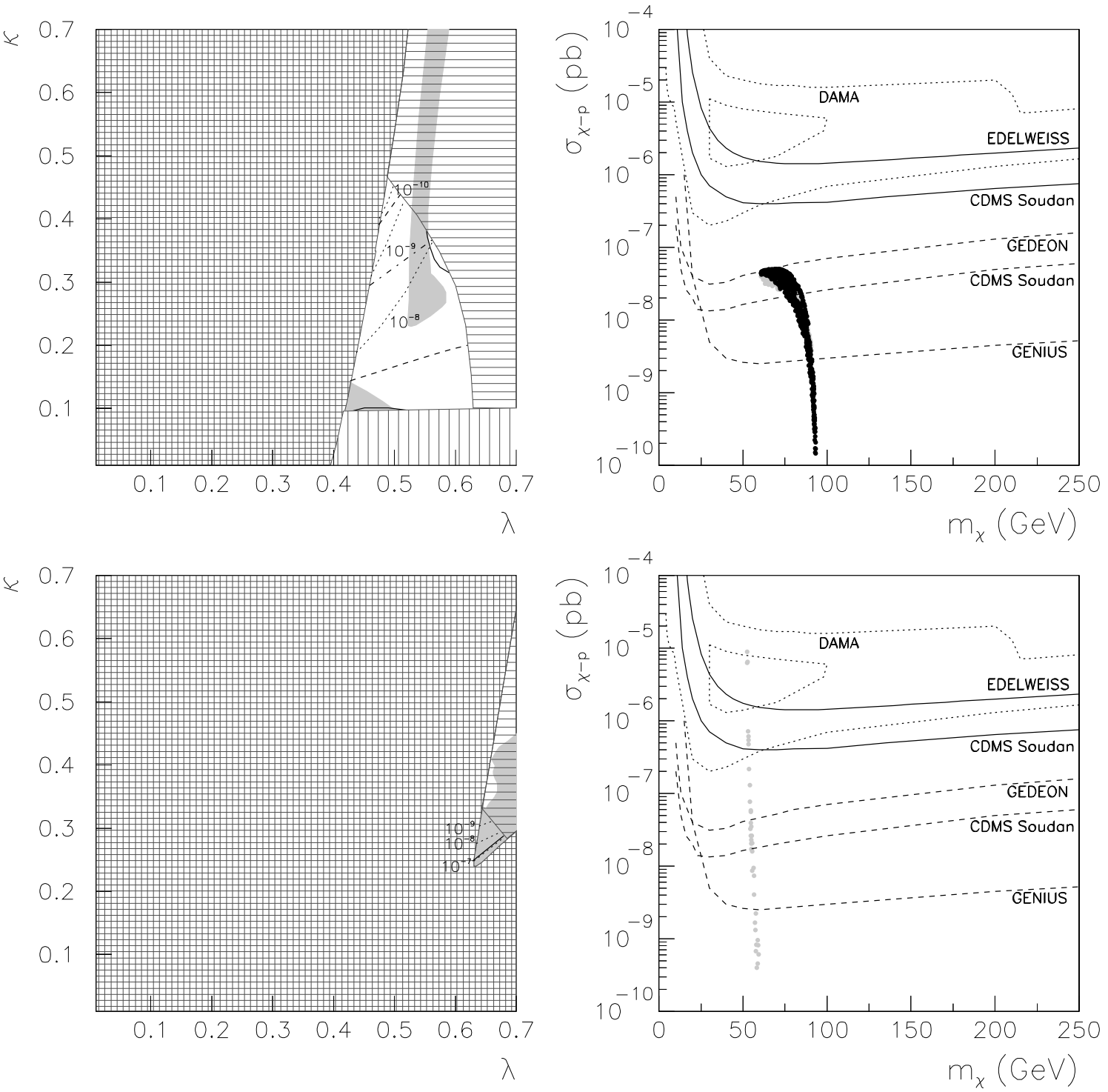

Figure 16: The same as in Fig. 4 but for the cases $A_{\lambda}=200 \mathrm{GeV}, \mu=110 \mathrm{GeV}, A_{\kappa}=$ $50 \mathrm{GeV}$, and $\tan \beta=2,5$, from top to bottom. In the case with $\tan \beta=2$ only the lines with $m_{h_{1}^{0}}=114,75 \mathrm{GeV}$, and those with $S_{13}^{2}=0.1$ and $N_{15}^{2}=0.1$ are represented. Similarly, in the case with $\tan \beta=5$ only the line with $m_{h_{1}^{0}}=25 \mathrm{GeV}$ is drawn, and none of the lines showing the compositions of the lightest scalar Higgs and neutralino is depicted, since $0.7>S_{13}^{2}>0.1$ and $0.4>N_{15}^{2}>0.1$ in all the plane.

parameter space is in general excluded.

We should now address the complementary choice of the sign of the parameters, 
namely $\mu, A_{\lambda}, A_{\kappa}<0$, for which we already know that the analysis of the Higgs sector still holds. Once more, differences arise in the theoretical predictions for $\sigma_{\tilde{\chi}_{1}^{0}-p}$.

To sum up, the choices for the signs of the parameters which have been considered in this section also permit obtaining large values for the theoretical prediction of the neutralino-nucleon cross section, despite the fact that the parameter space is very constrained both experimentally and by the occurrence of tachyons. In particular, values of $\sigma_{\tilde{\chi}_{1}^{0}-p}$ close to the sensitivities of the present detectors can be found in some regions of the parameter space. The lightest neutralino displays a mixed singlinoHiggsino character, and the scalar Higgs is singlet-like and light in those regions with larger $\sigma_{\tilde{\chi}_{1}^{0}-p}$.

\section{$4.4 \mu A_{\kappa}>0$ and $\mu A_{\lambda}>0(\kappa<0)$}

We will now focus our attention on those cases with a negative value for $\kappa$, namely $\mu, A_{\lambda}, A_{\kappa}>0$ and $\mu, A_{\lambda}, A_{\kappa}<0$.

Let us therefore concentrate on the first of the two possibilities, $\mu, A_{\lambda}, A_{\kappa}>0$. The parameter space is in this case plagued with tachyons in both the CP-even and $\mathrm{CP}$-odd Higgs sectors. On the one hand, regarding the CP-odd Higgses, large values of $|\kappa|$ and $\mu$ and small values of $\lambda$ and $A_{\lambda}$ may lead to negative values in the diagonal terms of the mass matrix, especially in $\mathcal{M}_{P, 11}^{2}$. Moreover, large values of $\lambda$ can also induce very large off-diagonal terms $\mathcal{M}_{P, 12}^{2}$ if $A_{\lambda}$ is large and a negative eigenvalue can be obtained in that case. Similar arguments lead to analogous conclusions concerning tachyons in the scalar sector, being the region with small $\lambda$ the one facing the most severe restrictions.

Note that experimental constraints will play a very important role in the vicinity of these regions. Although the largest exclusions typically arise from the bounds on IHDM and DHDM (mainly in $h^{0} \rightarrow b \bar{b}, h^{0} \rightarrow 2$ jets), APM can also exclude some regions with a small $m_{a_{1}^{0}}$. All these become particularly restrictive in the low $\tan \beta$ regime. In fact, in most of the cases with $\tan \beta \lesssim 3$ all of the parameter space is excluded. Experimental constraints in the neutralino sector can also be very stringent, especially for small values of $\mu$, where $\tilde{\chi}_{1}^{0}$ is light and Higgsino-like.

In the remaining allowed regions of the parameter space, the lightest CP-even Higgs is mostly dominated by the doublet component. Concerning the lightest neutralino, it turns out to be Higgsino-like. Owing to this, the predictions for $\sigma_{\tilde{\chi}_{1}^{0}-p}$ are very similar 
to those obtained in Section 4.2.

The above discussion can be illustrated with Fig. 17, where the $(\lambda, \kappa)$ plane and the predictions for $\sigma_{\tilde{\chi}_{1}^{0}-p}$ are presented for a case with $A_{\lambda}=450 \mathrm{GeV}, \mu=200 \mathrm{GeV}$, $A_{\kappa}=50 \mathrm{GeV}$, and $\tan \beta=5$. Light singlino-like neutralinos can only be obtained in the very small area with $|\kappa| \lesssim 0.06$, where the scalar Higgs may be as light as $m_{h_{1}^{0}} \gtrsim 50 \mathrm{GeV}$ with a large singlet component. In this particular region the predicted values for the detection cross section are not large, $\sigma_{\tilde{\chi}_{1}^{0}-p} \lesssim 10^{-9} \mathrm{pb}$. In the rest of the parameter space $\tilde{\chi}_{1}^{0}$ is Higgsino-like, which implies $m_{\tilde{\chi}_{1}^{0}} \approx \mu$, and the lightest scalar Higgs is a doublet with $m_{h_{1}^{0}} \gtrsim 112 \mathrm{GeV}$. Slightly higher values for the cross section are obtained, which are bounded by the experimental constraints on the scalar Higgs at $\sigma_{\tilde{\chi}_{1}^{0}-p} \lesssim 6 \times 10^{-8} \mathrm{pb}$. This prediction can be slightly increased with larger values of $\tan \beta$. For instance, with $\tan \beta=10$ one finds $\sigma_{\tilde{\chi}_{1}^{0}-p} \lesssim 2 \times 10^{-7} \mathrm{pb}$. None of the above remarks concerning the masses and compositions of the lightest neutralino and scalar Higgs would change in this case.

Variations in the rest of the parameters are very constrained due to the extensive tachyonic regions and the strong experimental bounds, especially those associated to the bounds on IHDM and DHDM. This is, for instance, what happens when the value of $A_{\lambda}$ decreases. The very narrow region for small $|\kappa|$ where singlino-like neutralinos can be obtained is usually ruled out and the only surviving areas are those featuring heavy Higgsino-like neutralinos and doublet-like scalar Higgses. For this reason, the predictions for the detection cross section are always similar to those presented in Fig. 17. As an example of some of the most favourable results for the cross section that can be obtained, we show in Fig. 18 a case with $A_{\lambda}=200 \mathrm{GeV}, \mu=110 \mathrm{GeV}$, $A_{\kappa}=200 \mathrm{GeV}$, and $\tan \beta=10$, where the sensitivity of the CDMS Soudan experiment is almost reached.

Finally, concerning the case $\mu, A_{\lambda}, A_{\kappa}<0$, nothing changes in the analysis of the Higgs sector. Once again, the differences in the neutralino sector and the slight changes in the experimental constraints can induce variations in the predicted $\sigma_{\tilde{\chi}_{1}^{0}-p}$. Nevertheless, a similar global upper bound of $\sigma_{\tilde{\chi}_{1}^{0}-p} \lesssim 10^{-7}$ pb is obtained.

The examples analysed in this Subsection feature a lightest neutralino which is Higgsino-like in most of the parameter space, together with a doublet-like lightest scalar Higgs. The neutralino-nucleon cross section is bounded by experimental constraints on the Higgs sector at $\sigma_{\tilde{\chi}_{1}^{0}-p} \lesssim 2 \times 10^{-7} \mathrm{pb}$. Singlino-like neutralinos can only be obtained in extremely small regions of the parameter space and predict smaller cross sections, $\sigma_{\tilde{\chi}_{1}^{0}-p} \lesssim 2 \times 10^{-9} \mathrm{pb}$. 

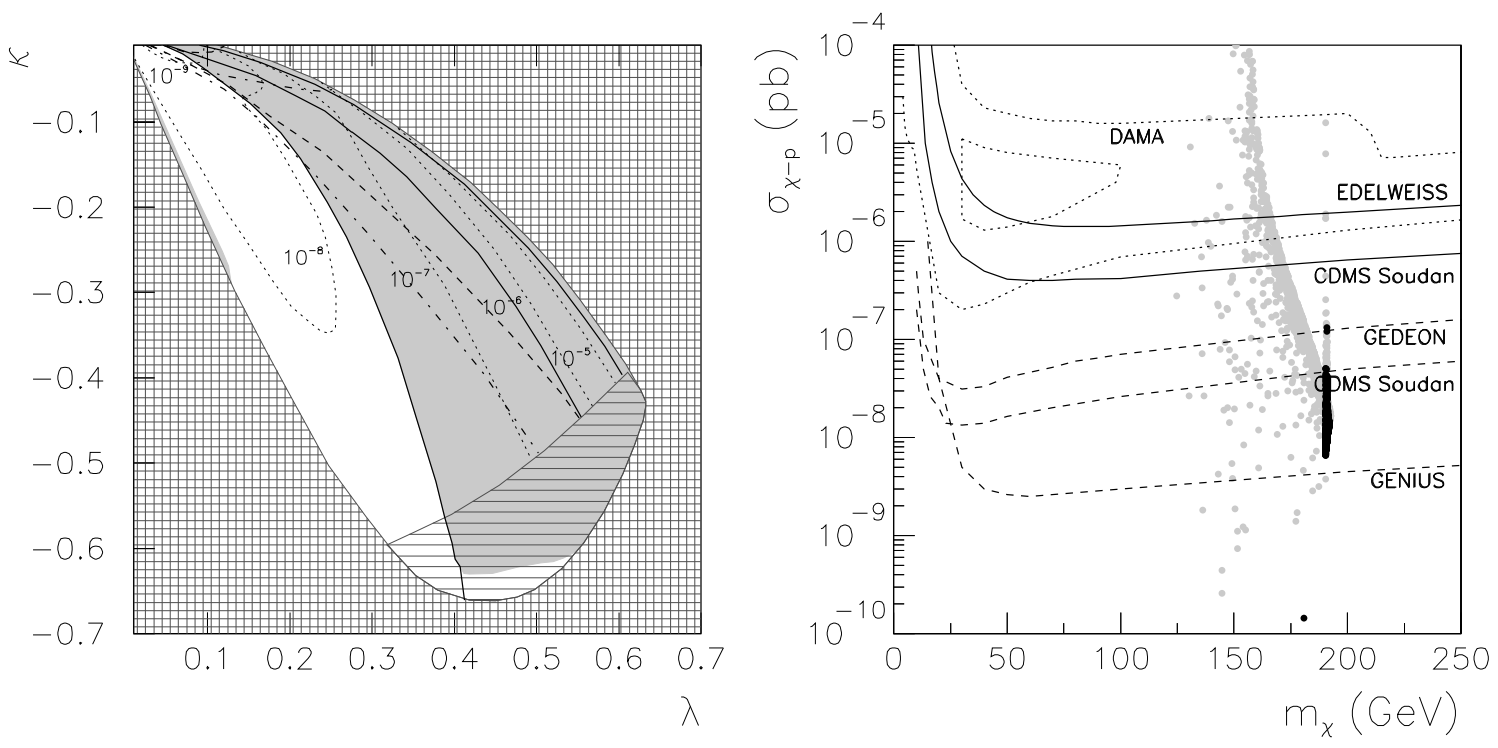

Figure 17: The same as in Fig. 4 but for negative $\kappa$, with $A_{\lambda}=450 \mathrm{GeV}, \mu=200 \mathrm{GeV}$, $A_{\kappa}=50 \mathrm{GeV}$ and $\tan \beta=5$.

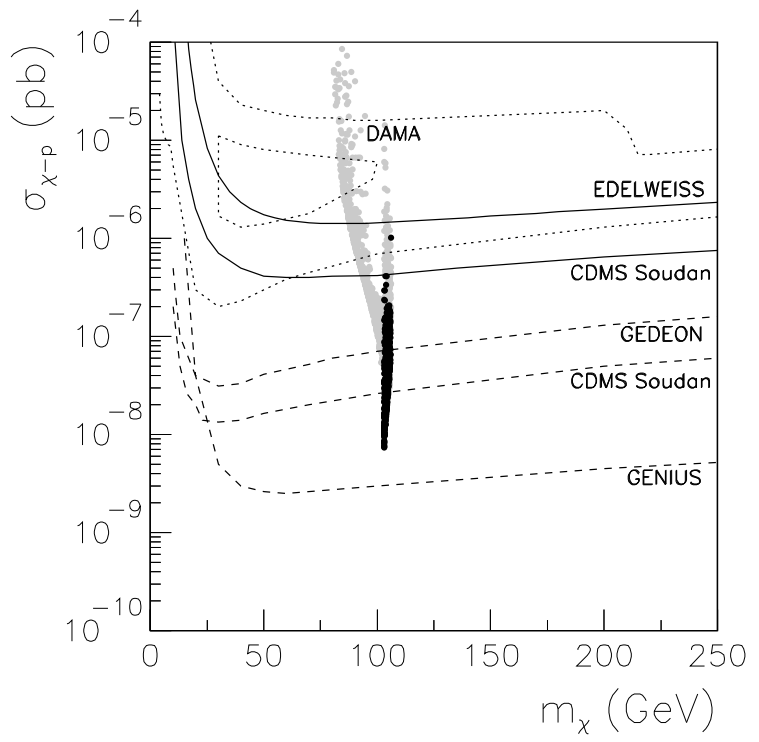

Figure 18: The same as in Fig. 17 but for $A_{\lambda}=200 \mathrm{GeV}, \mu=110 \mathrm{GeV}, A_{\kappa}=200 \mathrm{GeV}$ and $\tan \beta=10$. 


\subsection{Variations in the gaugino mass parameters}

In order to complete our analysis we must now address variations in the gaugino mass parameters. These clearly affect the neutralino sector, altering both the mass and composition of the lightest neutralino. In the former analysis we always assumed the relation $\mu<M_{1}<M_{2}$, which lead to neutralinos with important Higgsino compositions. We will now generalize our results for different hierarchies among these parameters. Namely, we will investigate the consequences of having $M_{1}<\mu, M_{2}$ or $M_{2}<\mu, M_{1}$.

The Higgs sector is not so sensitive to variations in the gaugino masses, since they only enter through loop corrections. For this reason, all the analysis regarding tachyons remains qualitatively valid, and the experimental constraints associated to Higgses exclude similar areas in all these cases. However, it is important to note that increasing the gaugino masses, especially the gluino mass, generally implies also an increase in the mass of the lightest scalar Higgs and for this reason, a heavy gaugino spectrum would typically lead to low values of $\sigma_{\tilde{\chi}_{1}^{0}-p}$.

Let us first vary the values of the gaugino masses preserving their GUT relation, but allowing also changes in the $\mu$ parameter so that the relation $M_{1}<\mu, M_{2}$ can be achieved, thus increasing the gaugino character of $\tilde{\chi}_{1}^{0}$. For this purpose we choose the example with $A_{\lambda}=200 \mathrm{GeV}, A_{\kappa}=-200 \mathrm{GeV}$, and $\tan \beta=3$ that was represented in Fig. 4, where now three different values for the $\mu$ parameter are taken, $\mu=110,200,500 \mathrm{GeV}$. Regarding the gaugino masses, we consider variations in the Bino mass as $50 \mathrm{GeV} \leq M_{1} \leq 500 \mathrm{GeV}$, and the GUT relation $M_{1}=\frac{1}{2} M_{2}=\frac{1}{7} M_{3}$. The results are shown in Fig. 19, where only those points fulfilling all the constraints are represented. Since the experimental constraint on the chargino mass imposes a lower bound on $M_{2}$, the value of the Bino mass is also constrained. For this reason, for low values of $\mu$ the lightest neutralino is still a singlino-Higgsino state $\left(N_{15}^{2} \lesssim 0.3\right.$ and $\left.N_{13}^{2}+N_{14}^{2} \gtrsim 0.7\right)$. However, as the value of $\mu$ increases, so does the gaugino composition of $\tilde{\chi}_{1}^{0}$ (note that both the Higgsino and singlino compositions decrease when increasing $\mu$ ). For instance, in the example with $\mu=500 \mathrm{GeV}$ neutralinos lighter than $m_{\tilde{\chi}_{1}^{0}} \lesssim 375 \mathrm{GeV}$ are Bino-like.

The appearance of Bino-like neutralinos has as a consequence the enormous decrease in the neutralino-nucleon cross section. In particular, the Higgs mediated interaction is now negligible and detection would only take place through the squark mediated interaction. In contrast to the huge predictions for $\sigma_{\tilde{\chi}_{1}^{0}-p}$ in the case of a singlino-Higgsino 

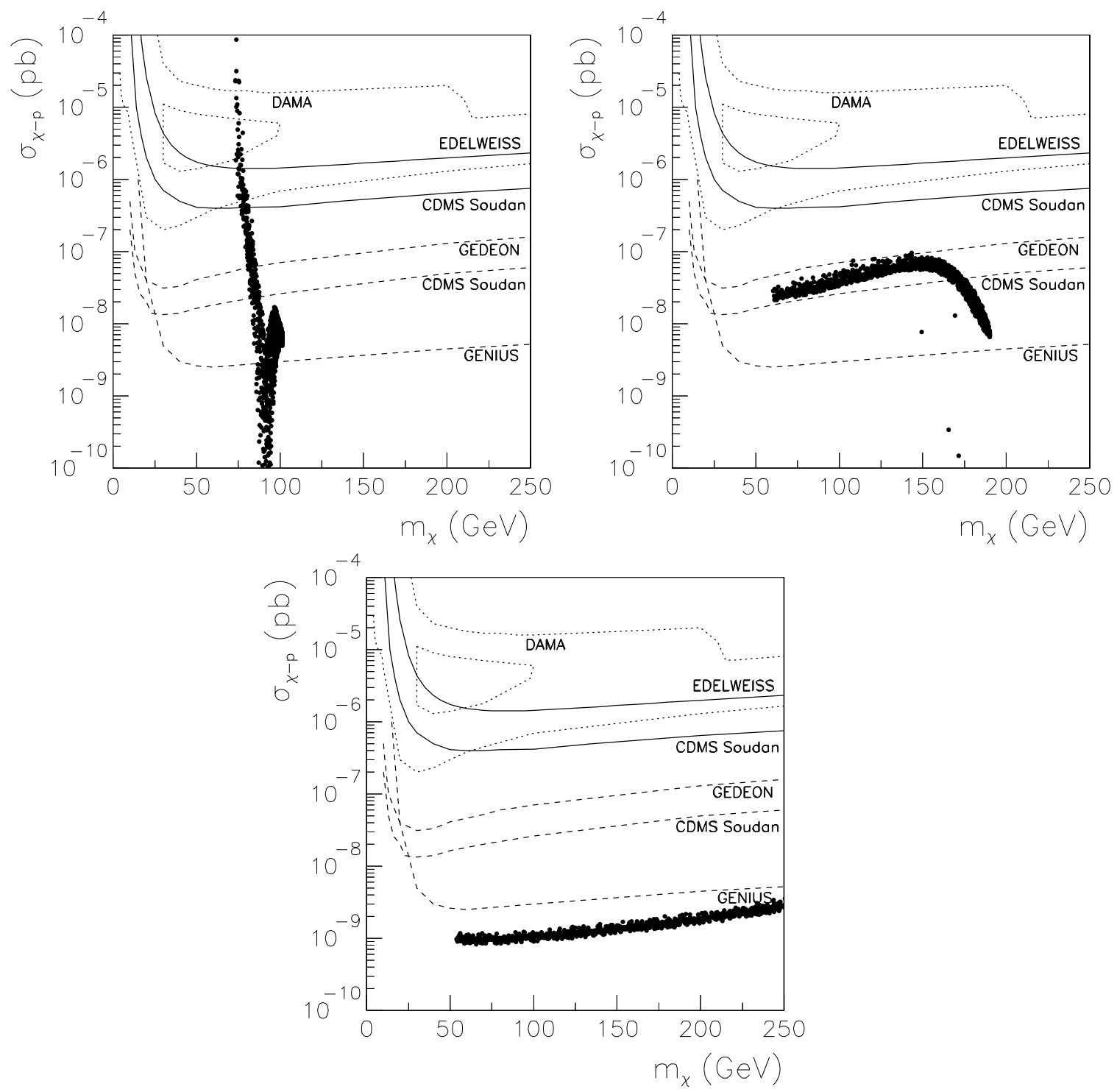

Figure 19: Scatter plot of the scalar neutralino-nucleon cross section as a function of the neutralino mass for $A_{\lambda}=200 \mathrm{GeV}, A_{\kappa}=-200 \mathrm{GeV}, \tan \beta=3, \mu=110,200,500 \mathrm{GeV}$ from left to right and from top to bottom. The gaugino masses satisfy the GUT relation and the gaugino mass parameter is varied in the range $50 \mathrm{GeV} \leq M_{1} \leq 500 \mathrm{GeV}$. Only those points fulfilling all the constraints are represented.

neutralino (see, e.g., the plot with $\mu=110 \mathrm{GeV}$ in Fig. 19), Bino-like neutralinos would have $\sigma_{\tilde{\chi}_{1}^{0}-p} \lesssim 10^{-9} \mathrm{pb}$, and thus would be beyond the sensitivities of even the largest projected dark matter detectors. 

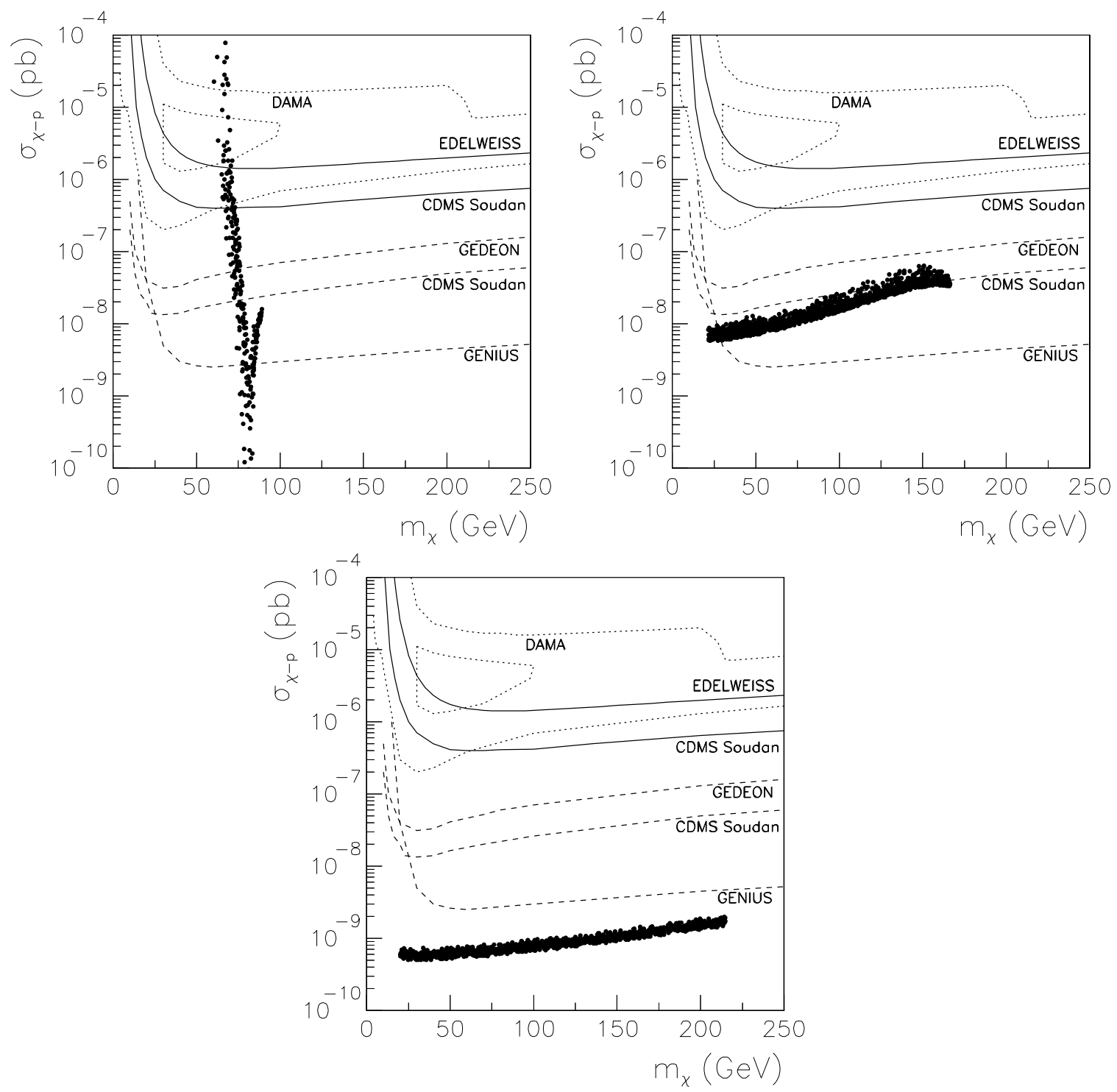

Figure 20: The same as Fig. 19 but for the relation $M_{1}=0.2 M_{2}$.

Very light Bino-like neutralinos can also appear when the GUT relation is relaxed. This is due to the freedom to choose very small values of $M_{1}$, unrelated to the more constrained (from the bound on the chargino mass) $M_{2}$. Furthermore, Bino-like neutralinos are not subject to such strong bounds on direct neutralino production as Higgsinolike neutralinos. In order to exemplify this possibility, we have chosen the relation $M_{1}=0.2 M_{2}$ (the GUT relation among the Wino and gluino masses, $M_{2}=\frac{2}{7} M_{3}$, is preserved) and applied it to the same examples as before. The results are represented in Fig. 20. Neutralinos as light as $m_{\tilde{\chi}_{1}^{0}} \gtrsim 25 \mathrm{GeV}$ are attainable, although the pre- 

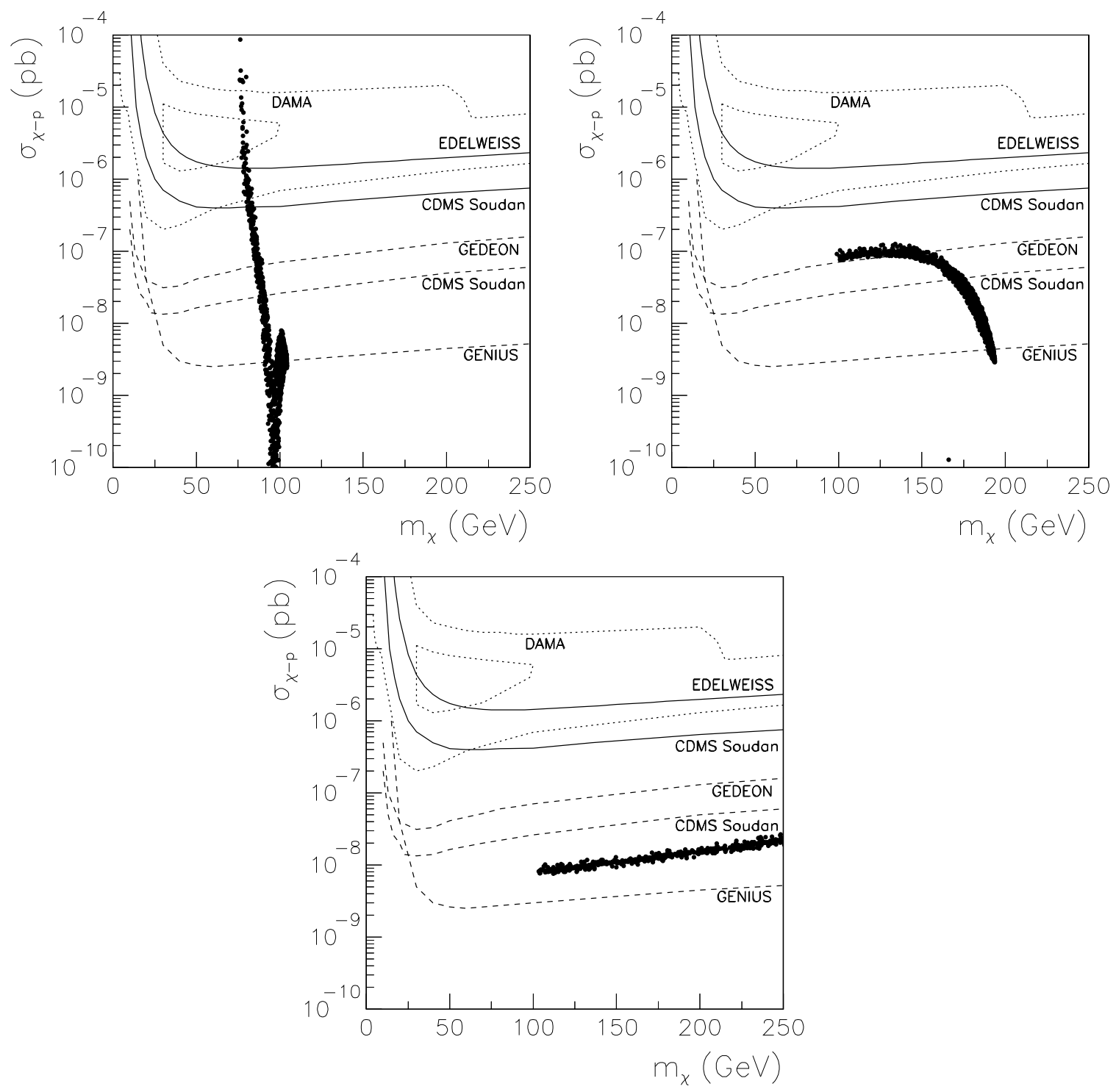

Figure 21: The same as Fig. 19 but for the relation $M_{1}=2 M_{2}=\frac{1}{7} M_{3}$.

dicted values for the detection cross section lie beyond the sensitivities of all present and projected experiments.

Finally, more general compositions for the neutralino can also be found. In particular, Wino-like neutralinos are attainable if $M_{2}<M_{1}, \mu$. This is an interesting possibility, since Wino-like neutralinos predict in general larger values for the cross section than Bino-like $\tilde{\chi}_{1}^{0}$. In order to explore this possibility we have repeated the same scan in the parameter space as above, but now with the relation $M_{1}=2 M_{2}=1 / 7 M_{3}$ for the gaug- 
ino masses at the EW scale. The results are shown in Fig. 21 for $\mu=110,200,500 \mathrm{GeV}$. Obviously, the Wino component of $\tilde{\chi}_{1}^{0}$ becomes more important for large values of $\mu$. Thus, for instance, whereas in the example with $\mu=200 \mathrm{GeV} \tilde{\chi}_{1}^{0}$ is a mixed Higgsino-Wino state (with $N_{12}^{2} \lesssim 0.6$ ), Wino-like neutralinos populate the example with $\mu=500 \mathrm{GeV}$. Because of the experimental constraint on the chargino mass, Wino-like neutralinos cannot be obtained below $m_{\tilde{\chi}_{1}^{0}} \approx 100 \mathrm{GeV}$. Note that although the theoretical predictions for the detection cross section are larger than in the case of Bino-like neutralinos of Fig. 19, there is an important decrease with respect to those in the case of Higgsino-singlino neutralinos. In particular, in this case $\sigma_{\tilde{\chi}_{1}^{0}-p} \lesssim 2 \times 10^{-8} \mathrm{pb}$.

All the variations discussed in this Subsection imply a variation in the mass and composition of the lightest neutralino and scalar Higgs. We have seen how Binoand Wino-like neutralinos are attainable by decreasing $M_{1}$ and $M_{2}$, respectively, and increasing $\mu$. The singlino-Higgsino character of the neutralino is therefore lost in these cases. Furthermore, the increase in $\mu$ also leads to doublet-like heavier Higgses. For these reasons the Higgs mediated interaction is no longer effective and the theoretical predictions for the neutralino-nucleon cross section can have a huge decrease. Therefore, the optimal situation is the one we have analysed in all the examples of Subsections. 4.1 to 4.4 , where $\mu$ is close to its lower accepted value and smaller than the gaugino masses.

\subsection{Overview}

In the previous Subsections we have presented a separate analysis of the distinct regions of the $A_{\lambda}, A_{\kappa}$, and $\tan \beta$ parameter space. This kind of approach was useful in order to comprehend the implications of individual variations of these parameters. Still, in order to obtain a global view on the theoretical predictions for $\sigma_{\tilde{\chi}_{1}^{0}-p}$, and their compatibility with present and projected dark matter detectors, it is useful to conduct a more general survey of the parameter space. Such an analysis constitutes a good overview of the properties of the cases studied in the previous Subsections.

Since we are interested in regions predicting large $\sigma_{\tilde{\chi}_{1}^{0}-p}$, according to the conclusions of Subsection 4.5 we will focus our attention on the case $\mu=110 \mathrm{GeV}$, with heavy gaugino masses, $M_{1}=\frac{1}{2} M_{2}=500 \mathrm{GeV}$. The rest of the input parameters are allowed to vary in the ranges, $-600 \mathrm{GeV} \leq A_{\lambda} \leq 600 \mathrm{GeV},-400 \mathrm{GeV} \leq A_{\kappa} \leq 400 \mathrm{GeV}$, and $\tan \beta=2,3,4,5,10$, with $\lambda,|\kappa| \in[0.01,0.8]$.

The results of this scan are shown in Figs. 22 and 23, for positive and negative $\kappa$, 

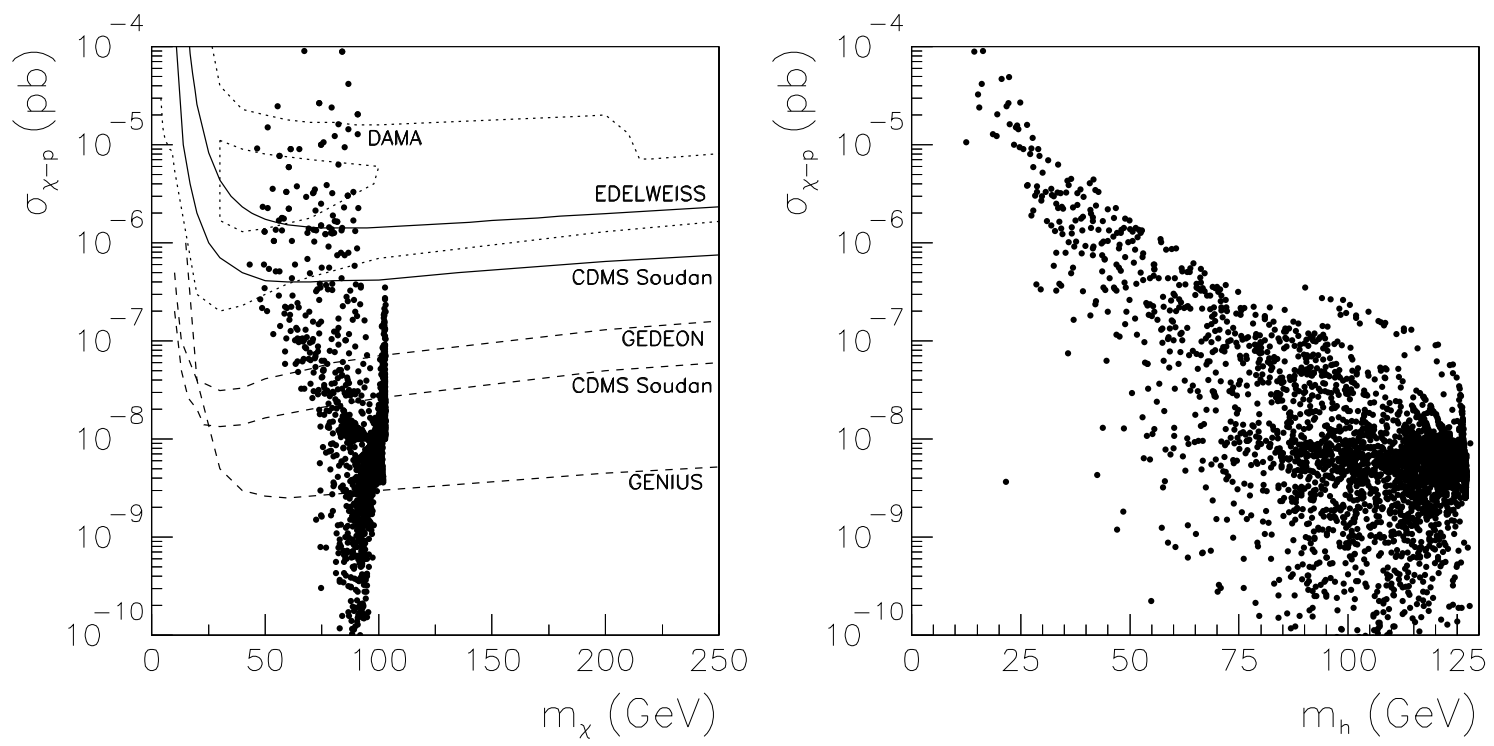

Figure 22: The same as in Fig. 3 but for $\mu=110 \mathrm{GeV}$ and the rest of the parameters in the ranges $-600 \mathrm{GeV} \leq A_{\lambda} \leq 600 \mathrm{GeV},-400 \mathrm{GeV} \leq A_{\kappa} \leq 400 \mathrm{GeV}$, and $\tan \beta=2,3,4,5,10$, and with positive values of $\kappa$. Only those points fulfilling all the constraints are represented.

respectively, where the theoretical predictions for $\sigma_{\tilde{\chi}_{1}^{0}-p}$ are represented as a function of the lightest neutralino and scalar Higgs masses. Fig. 22 therefore summarizes the results of Sections 4.1, 4.2, and 4.3. Points with large predictions for $\sigma_{\tilde{\chi}_{1}^{0}-p}$ are found. These correspond to very light singlet-like Higgses, with even $m_{h_{1}^{0}} \gtrsim 15 \mathrm{GeV}$, which are more easily obtained for low values of $\tan \beta(\tan \beta \lesssim 5)$. The points in this region correspond to those with $\mu A_{\lambda}>0$, discussed in Sections 4.1 and 4.3, respectively. The lightest neutralino in those points has an important singlino composition, $N_{15}^{2} \lesssim 0.6$.

Fig. 23 generalizes the analysis of Section 4.4. As we had already discussed there, the lightest scalar Higgs is heavier, $m_{h_{1}^{0}} \gtrsim 75 \mathrm{GeV}$, and although it can be singlet-like in some cases, the predictions for the neutralino-nucleon cross section are typically low. Regarding the neutralino, it is Higgsino-like in most of the parameter space and therefore $m_{\tilde{\chi}_{1}^{0}} \approx \mu$. 

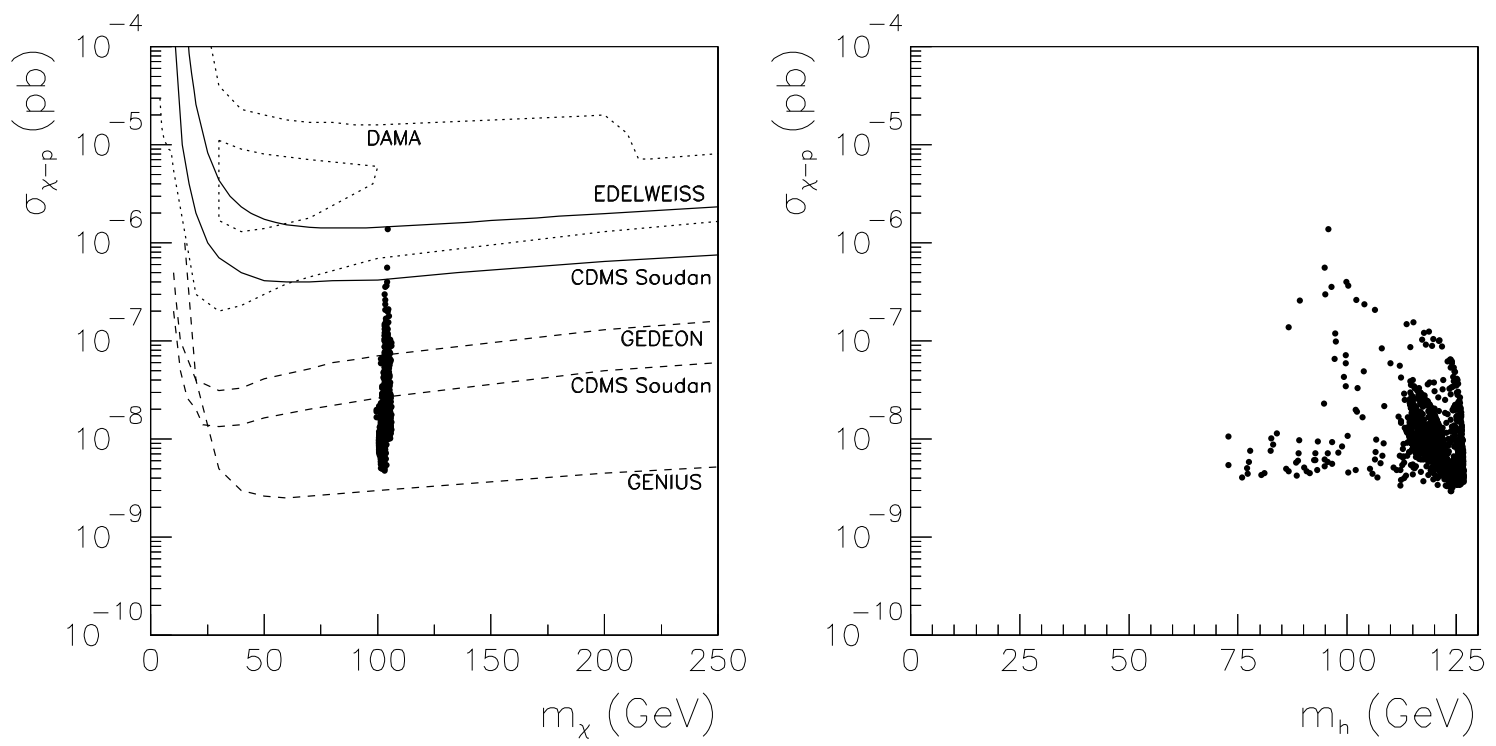

Figure 23: The same as in Fig. 22 but for negative values of $\kappa$.

\section{Conclusions}

We have performed a systematic analysis of the low-energy parameter space of the Nextto-Minimal Supersymmetric Standard Model (NMSSM), studying the implications for the direct detection of neutralino dark matter. We have thus computed the theoretical predictions for the scalar neutralino-proton cross section, $\sigma_{\tilde{\chi}_{1}^{0}-p}$, and compared it with the sensitivities of present and projected dark matter experiments. In the computation we have taken into account all available experimental constraints from LEP on the parameter space.

We have found that large values of $\sigma_{\tilde{\chi}_{1}^{0}-p}$, even within the reach of present dark matter detectors (see e.g. Fig. 22), can be obtained in regions of the parameter space. This is essentially due to the exchange of very light Higgses, $m_{h_{1}^{0}} \lesssim 70 \mathrm{GeV}$. The NMSSM nature is evidenced in this result, since such Higgses have a significant singlet composition, thus escaping detection and being in agreement with accelerator data. In fact, Higgses as light as $15 \mathrm{GeV}$ can be obtained. The lightest neutralino in those regions exhibits a large singlino-Higgsino composition, and a mass in the range $50 \lesssim m_{\tilde{\chi}_{1}^{0}} \lesssim 100 \mathrm{GeV}$.

Let us finally mention that our work can be considered as a first step towards a more complete analysis of the direct detection of neutralino dark matter in the NMSSM. As discussed in Sections 2.5 and 4, other potentially important constraints 
on the parameter space should be addressed in forthcoming publications. This is the case e.g. of the relic density, the $b \rightarrow s \gamma$ branching ratio, and the current upper limit on the decay $B_{s} \rightarrow \mu^{+} \mu^{-}$.

\section{Acknowledgements}

The work of D.G. Cerdeño was supported in part by the Deutsche Forschungsgemeinschaft, the DAAD, and the European Union under contract HPRN-CT-2000-00148. C. Hugonie was supported by the European Union RTN grant HPRN-CT-2000-00148. D.E. López-Fogliani acknowledges the financial support of the Spanish DGI through a FPU grant, and also through the Acción Integrada Hispano-Alemana HA2002-0117. The work of C. Muñoz was supported in part by the Spanish DGI under Acción Integrada Hispano-Alemana HA2002-0117, and under contracts BFM2003-01266 and FPA2003-04597, and also by the European Union under contract HPRN-CT-200000148. A.M. Teixeira acknowledges the support by Fundação para a Ciência e Tecnologia under the grant SFRH/BPD/11509/2002, and also by the Spanish DGI under Acción Integrada Hispano-Alemana HA2002-0117 and under contract BFM2003-01266. D.G. Cerdeño and A.M. Teixeira are also thankful to the organizers of the workshop "Terrestrial and Cosmic Neutrinos, Leptogenesis and Cosmology" in the Benasque Center for Science for their hospitality.

\section{A Relevant NMSSM interaction vertices}

\section{A.1 Higgs-quark-quark Yukawa coupling}

Parameterising the interaction of neutral CP-even Higgs fields with quarks as

$$
\mathcal{L}_{q q h}=-\bar{q}_{i}\left[C_{Y L}^{i} P_{L}+C_{Y R}^{i} P_{R}\right] q_{i} h_{a}^{0},
$$

where $h_{a}^{0}$ denotes the physical (mass) Higgs eigenstates and $i=1,2$ up- and down-type quarks, one has

$$
C_{Y L}^{i}=C_{Y R}^{i}=C_{Y}^{i} ; \quad C_{Y}^{1}=-\frac{g m_{u}}{2 M_{W} \sin \beta} S_{a 2}, \quad C_{Y}^{2}=-\frac{g m_{d}}{2 M_{W} \cos \beta} S_{a 1}
$$

where we have omitted the quark generations and $S$ is the unitary matrix that diagonalises the scalar Higgs mass matrix, defined in Eq. (2.9). 


\section{A.2 Neutralino-neutralino-Higgs interaction}

The interaction of scalar Higgs and neutralinos can be parametrised as

$$
\mathcal{L}_{h \tilde{\chi}^{0} \tilde{\chi}^{0}}=\frac{1}{4} h_{a}^{0} \overline{\tilde{\chi}}_{\alpha}^{0}\left[C_{H L}^{a \alpha \beta} P_{L}+C_{H R}^{a \alpha \beta} P_{R}\right] \tilde{\chi}_{\beta}^{0},
$$

where $a=1-3$ refers to the Higgs mass eigenstate, $\alpha, \beta=1-5$ denote the physical neutralino states, and the couplings are defined as

$$
\begin{aligned}
C_{H L}^{a \alpha \beta}= & \left\{-g\left(N_{\alpha 2}^{*}-\tan \theta_{W} N_{\alpha 1}^{*}\right)\left(S_{a 1} N_{\beta 3}^{*}-S_{a 2} N_{\beta 4}^{*}\right)+\right. \\
& \left.+\sqrt{2} \lambda\left[S_{a 3} N_{\alpha 3}^{*} N_{\beta 4}^{*}+N_{\beta 5}^{*}\left(S_{a 2} N_{\alpha 3}^{*}+S_{a 1} N_{\alpha 4}^{*}\right)\right]+(\alpha \rightarrow \beta)\right\} \\
& -2 \sqrt{2} \kappa S_{a 3} N_{\alpha 5}^{*} N_{\beta 5}^{*}, \\
C_{H R}^{a \alpha \beta}= & \left(C_{H L}^{a \alpha \beta}\right)^{*} .
\end{aligned}
$$

In the text, and since we have exclusively analysed interactions involving the lightest neutralino states (i.e. $\alpha=\beta=1$ ), we have simplified the above as $C_{H L}^{a 11}=C_{H L}^{a}$ and $C_{H R}^{a 11}=C_{H R}^{a}$.

\section{A.3 Neutralino-squark-quark interaction}

In terms of the mass eigenstates, the Lagrangian reads

$$
\mathcal{L}_{q \tilde{q} \tilde{\chi}^{0}}=\bar{q}_{i}\left[C_{L}^{\alpha X i} P_{L}+C_{R}^{\alpha X i} P_{R}\right] \tilde{\chi}_{\alpha}^{0} \tilde{q}_{i}^{X},
$$

where $i=1,2$ denotes an up- or down-type quark and squark, $X=1,2$ the squark mass eigenstates, and $\alpha=1, \ldots, 5$ the neutralino states. Since we have neglected flavour violation in the squark sector, only $L R$ mixing occurs, and squark physical and chiral eigenstates are related as

$$
\left(\begin{array}{c}
\tilde{q}_{1} \\
\tilde{q}_{2}
\end{array}\right)=\left(\begin{array}{cc}
\eta_{11}^{\tilde{q}} & \eta_{12}^{\tilde{q}} \\
\eta_{21}^{\tilde{q}} & \eta_{22}^{\tilde{q}}
\end{array}\right)\left(\begin{array}{c}
\tilde{q}_{L} \\
\tilde{q}_{R}
\end{array}\right) .
$$

One can also make the usual redefinition $\eta_{11}^{\tilde{q}}=\eta_{22}^{\tilde{q}}=\cos \theta_{\tilde{q}}$ and $\eta_{12}^{\tilde{q}}=-\eta_{21}^{\tilde{q}}=\sin \theta_{\tilde{q}}$ Therefore, for the up sector, and again omitting quark and squark generation indices, 
the coefficients $C_{L, R}^{\alpha X i}$ are given by:

$$
\begin{aligned}
& C_{L}^{\alpha 11}=-\sqrt{2} g\left[\frac{Y_{u}}{2} \tan \theta_{W} N_{\alpha 1}^{*} \sin \theta_{\tilde{u}}+\frac{m_{u}}{2 M_{W} \sin \beta} N_{\alpha 4}^{*} \cos \theta_{\tilde{u}}\right], \\
& C_{R}^{\alpha 11}=-\sqrt{2} g\left\{\left[N_{\alpha 2}^{*} T_{3}^{u}+\frac{Y_{Q}}{2} \tan \theta_{W} N_{\alpha 1}^{*}\right] \cos \theta_{\tilde{u}}+\frac{m_{u}}{2 M_{W} \sin \beta} N_{\alpha 4}^{*} \sin \theta_{\tilde{u}}\right\}, \\
& C_{L}^{\alpha 21}=-\sqrt{2} g\left[\frac{Y_{u}}{2} \tan \theta_{W} N_{\alpha 1}^{*} \cos \theta_{\tilde{u}}-\frac{m_{u}}{2 M_{W} \sin \beta} N_{\alpha 4}^{*} \sin \theta_{\tilde{u}}\right], \\
& C_{R}^{\alpha 21}=-\sqrt{2} g\left\{\left[-N_{\alpha 2}^{*} T_{3}^{u}+\frac{Y_{Q}}{2} \tan \theta_{W} N_{\alpha 1}^{*}\right] \sin \theta_{\tilde{u}}+\frac{m_{u}}{2 M_{W} \sin \beta} N_{\alpha 4}^{*} \cos \theta_{\tilde{u}}\right\} .
\end{aligned}
$$

In the above, $Y_{Q(u)}$ denotes the hypercharge of the $S U(2)_{L}$ quark doublet (up-singlet) and $T_{3}^{u}$ the isospin of the $u_{L}$ field. The analogous for the down sector is trivially obtained by the appropriate replacements $\left(Y_{u} \rightarrow Y_{d}, T_{3}^{u} \rightarrow T_{3}^{d}, m_{u} \rightarrow m_{d}, \theta_{\tilde{u}} \rightarrow \theta_{\tilde{d}}\right.$, $\sin \beta \rightarrow \cos \beta$ and $\left.N_{\alpha 4} \rightarrow N_{\alpha 3}\right)$. In this paper, and since only $q-\tilde{q}-\tilde{\chi}_{1}^{0}$ interactions

have been considered, we have always used $C_{L, R}^{1 X i}=C_{L, R}^{X i}$, i.e., setting $\alpha=1$ in the above.

\section{References}

[1] For a recent review see, C. Muñoz, 'Dark matter detection in the light of recent experimental results', to appear in Int. J. Mod. Phys. A [arXiv:hep-ph/0309346].

[2] DAMA Collaboration, R. Bernabei et al., 'Search for WIMP annual modulation signature: results from DAMA/NaI-3 and DAMA/NaI-4 and the global combined analysis', Phys. Lett. B480 (2000) 23; 'Dark matter search', Riv. N. Cim. 26 (2003) 1 [arXiv:astro-ph/0307403].

[3] P. Belli, R. Cerulli, N. Fornengo and S. Scopel, 'Effect of the galactic halo modeling on the DAMA/NaI annual modulation result: an extended analysis of the data for WIMPs with a purely spin-independent coupling', Phys. Rev. D66 (2002) 043503 [arXiv:hep-ph/0203242].

[4] CDMS Collaboration, D.S. Akerib et al., 'First results from the cryogenic dark matter search in the Soudan underground lab', arXiv:hep-ph/0405033.

[5] EDELWEISS Collaboration, A. Benoit et al., 'First results of the EDELWEISS WIMP search using a 320-g heat-and-ionization Ge detector', Phys. Lett. B513 
(2001) 15 [arXiv:astro-ph/0106094]; 'Improved exclusion limits from the EDELWEISS WIMP search', Phys. Lett. B545 (2002) 43 [arXiv:astro-ph/0206271].

[6] ZEPLIN I Collaboration, N.J.T. Smith et al., talk given at TAUP 2003, Seattle, Washington.

[7] P. Ullio, M. Kamionkowski and P. Vogel, 'Spin-dependent WIMPs in DAMA?', J. High Energy Phys. 07 (2001) 044 [arXiv:hep-ph/0010036];

D. Smith and N. Weiner, 'Inelastic dark matter', Phys. Rev. D64 (2001) 043502 [arXiv:hep-ph/0101138]; 'Inelastic dark matter at DAMA, CDMS and future experiments', talk given at DM 2002 Conference, Marina del Rey, California (2002), arXiv:astro-ph/0208403;

R. Bernabei et al., 'Investigating the DAMA annual modulation data in the framework of inelastic dark matter', Eur. Phys. J. C23 (2002) 61;

G. Prezeau, A. Kurylov, M. Kamionkowski and P. Vogel, 'New contribution to WIMP-nucleus scattering', Phys. Rev. Lett. 91 (2003) 231301 [arXiV:astro$\mathrm{ph} / 0309115]$;

C. Savage, P. Gondolo and K. Freese, 'Can WIMP spin dependent couplings explain DAMA data, in the light of null results from other experiments?', arXiv:astro-ph/0408346.

[8] A. Morales, 'Searching for WIMP dark matter: the case for Germanium ionization detectors', talk given at the 29th International Meeting on Fundamental Physics, Sitges, Barcelona (2001), arXiv:hep-ex/0111089.

[9] GENIUS Collaboration, H.V. Klapdor-Kleingrothaus et al., 'GENIUS - a Supersensitive Germanium Detector System for Rare Events', arXiv:hep-ph/9910205.

[10] See e.g., E. Aprile et al., 'The XENON dark matter search experiment', arXiv:astro-ph/0407575.

[11] H. Goldberg, 'Constraint on the photino mass from cosmology', Phys. Rev. Lett. 50 (1983) 1419;

J. Ellis, J.S. Hagelin, D.V. Nanopoulos and M. Srednicki, 'Search for supersymmetry at the $\bar{p} p$ collider', Phys. Lett. B127 (1983) 233;

L.M. Krauss "New constraints on "INO" masses from cosmology (I). Supersymmetric "inos"', Nucl. Phys. B227 (1983) 556; 
J. Ellis, J.S. Hagelin, D.V. Nanopoulos, K.A. Olive and M. Srednicki, 'Supersymmetric relics from the Big Bang', Nucl. Phys. B238 (1984) 453.

[12] J.E. Kim and H.P. Nilles, 'The $\mu$ problem and the strong CP problem' Phys. Lett. B138 (1984) 150.

[13] G.F. Giudice and A. Masiero, 'A natural solution to the $\mu$ problem in supergravity theories', Phys. Lett. B206 (1988) 480;

J.E. Kim and H.P. Nilles, Gaugino condensation and the cosmological implications of the hidden sector', Phys. Lett. B263 (1991) 79;

E.J. Chun, J.E. Kim and H.P. Nilles, 'A natural solution of the $\mu$ problem with a composite axion in the hidden sector', Nucl. Phys. B370 (1992) 105;

J.A. Casas and C. Muñoz, 'A natural solution to the $\mu$ problem', Phys. Lett. B306 (1993) 288 [arXiv:hep-ph/9302227];

G. Lopes-Cardoso, D. Lüst and T. Mohaupt, 'Moduli spaces and target space duality symmetries in $(0,2) Z_{n}$ orbifold theories with continuous Wilson lines', Nucl. Phys. B432 (1994) 68 [arXiv:hep-th/9405002];

I. Antoniadis, E. Gava, K.S. Narain and T.R. Taylor, 'Effective $\mu$ term in superstring theory', Nucl. Phys. B432 (1994) 187 [arXiv:hep-th/9405024];

A. Brignole, L.E. Ibáñez and C. Muñoz, 'Orbifold-induced $\mu$ term and electroweak symmetry breaking', Phys. Lett. B387 (1996) 769 [arXiv:hep-ph/9607405];

K. Choi, J.S. Lee and C. Muñoz, 'Supergravity radiative effects on soft terms and the $\mu$ term', Phys. Rev. Lett. 80 (1998) 3686 [arXiv:hep-ph/9709250].

[14] H. P. Nilles, M. Srednicki and D. Wyler, 'Weak interaction breakdown induced by supergravity', Phys. Lett. B120 (1983) 346;

J. M. Frere, D. R. T. Jones and S. Raby, 'Fermion masses and induction of the weak scale by supergravity', Nucl. Phys. B222 (1983) 11;

J. P. Derendinger and C. A. Savoy, 'Quantum effects and SU(2) X U(1) breaking in supergravity gauge theories', Nucl. Phys. B237 (1984) 307.

[15] J. R. Ellis, J. F. Gunion, H. E. Haber, L. Roszkowski and F. Zwirner, 'Higgs bosons in a nonminimal supersymmetric model', Phys. Rev. D39 (1989) 844; M. Drees, 'Supersymmetric models with extended Higgs sector', Int. J. Mod. Phys. A4 (1989) 3635;

U. Ellwanger, M. Rausch de Traubenberg and C. A. Savoy, 'Particle spectrum in supersymmetric models with a gauge singlet', Phys. Lett. B315 (1993) 331 
[arXiv:hep-ph/9307322]; 'Phenomenology of supersymmetric models with a singlet', Nucl. Phys. B492 (1997) 21 [arXiv:hep-ph/9611251];

S. F. King and P. L. White, 'Resolving the constrained minimal and nextto-minimal supersymmetric standard models', Phys. Rev. D52 (1995) 4183 [arXiv:hep-ph/9505326].

[16] M. Bastero-Gil, C. Hugonie, S. F. King, D. P. Roy and S. Vempati, 'Does LEP prefer the NMSSM?', Phys. Lett. B489 (2000) 359 [arXiv:hep-ph/0006198].

[17] S. A. Abel, S. Sarkar and P. L. White, 'On the cosmological domain wall problem for the minimally extended supersymmetric standard model', Nucl. Phys. B454 (1995) 663 [arXiv:hep-ph/9506359].

[18] S. A. Abel, 'Destabilising divergences in the NMSSM', Nucl. Phys. B480 (1996) 55 [arXiv:hep-ph/9609323];

C. Panagiotakopoulos and K. Tamvakis, 'Stabilized NMSSM without domain walls', Phys. Lett. B446 (1999) 224 [arXiv:hep-ph/9809475].

[19] U. Ellwanger and C. Hugonie, 'Topologies of the $(\mathrm{M}+1) \mathrm{SSM}$ with a singlino LSP at LEP2', Eur. Phys. J. C13 (2000) 681 [arXiv:hep-ph/9812427].

[20] U. Ellwanger and C. Hugonie, 'Masses and couplings of the lightest Higgs bosons in the (M+1)SSM', Eur. Phys. J. C25 (2002) 297 [arXiv:hep-ph/9909260].

[21] U. Ellwanger, J. F. Gunion and C. Hugonie, 'NMHDECAY: A Fortran code for the Higgs masses, couplings and decay widths in the NMSSM', arXiv:hep-ph/0406215.

[22] U. Ellwanger, J. F. Gunion, C. Hugonie and S. Moretti, 'NMSSM Higgs discovery at the LHC', arXiv:hep-ph/0401228.

[23] R. Flores, K.A. Olive and D. Thomas, 'Light-neutralino interactions in matter in an extended supersymmetric standard model', Phys. Lett. B263 (1991) 425.

[24] V.A. Bednyakov and H.V. Klapdor-Kleingrothaus, 'About direct dark matter detection in next-to-minimal supersymmetric standard model', Phys. Rev. D59 (1999) 023514 [arXiv:hep-ph/9802344].

[25] B.R. Greene and P.J. Miron, 'Supersymmetric cosmology with a gauge singlet', Phys. Lett. B168 (1986) 226;

R. Flores, K.A. Olive and D. Thomas, 'A new dark matter candidate in the minimal extension of the supersymmetric standard model', Phys. Lett. B245 (1990) 
509 ;

K.A. Olive and D. Thomas, 'A light dark matter candidate in an extended supersymmetric model', Nucl. Phys. B355 (1991) 192;

S.A. Abel, S. Sarkar and I.B. Whittingham, 'Neutralino dark matter in a class of unified theories', Nucl. Phys. B392 (1993) 83 [arXiv:hep-ph/9209292];

A. Stephan, 'Dark matter constraints on the parameter space and particle spectra in the nonminimal SUSY standard model', Phys. Lett. B411 (1997) 97 [arXiv:hepph/9704232]; 'Dark matter constraints in the minimal and nonminimal SUSY standard model', Phys. Rev. D58 (1998) 035011 [arXiv:hep-ph/9709262];

A. Menon, D. E. Morrissey and C. E. M. Wagner, 'Electroweak baryogenesis and dark matter in the nMSSM', arXiv:hep-ph/0404184.

[26] H. P. Nilles, M. Srednicki and D. Wyler, 'Constraints on the stability of mass hierarchies in supergravity', Phys. Lett. B124 (1983) 337;

U. Ellwanger, 'Nonrenormalizable interactions from supergravity, quantum corrections and effective low-energy theories', Phys. Lett. B133 (1983) 187;

J. Bagger and E. Poppitz, 'Destabilizing divergences in supergravity coupled supersymmetric theories', Phys. Rev. Lett. 71 (1993) 2380 [arXiv:hep-ph/9307317]; J. Bagger, E. Poppitz and L. Randall, 'Destabilizing divergences in supergravity theories at two loops', Nucl. Phys. B455 (1995) 59 [arXiv:hep-ph/9505244].

[27] J. C. Romao, 'Spontaneous CP violation in susy models: a no go theorem', Phys. Lett. B173 (1986) 309.

[28] K. Hagiwara et al., Phys. Rev. D66 (2002) 010001.

[29] DELPHI Collaboration, J. Abdallah et al., 'Searches for supersymmetric particles in e+ e- collisions up to $208-\mathrm{GeV}$ and interpretation of the results within the MSSM', Eur. Phys. J. C31 (2004) 421 [arXiv:hep-ex/0311019];

OPAL Collaboration, G. Abbiendi et al., 'Search for chargino and neutralino production at $\mathrm{s}^{* *}(1 / 2)=192-\mathrm{GeV}-209-\mathrm{GeV}$ at LEP', Eur. Phys. J. C35 (2004) 1 [arXiv:hep-ex/0401026].

[30] LEP SUSY Working Group, LEPSUSYWG Note/02-04.1.

[31] LEP Higgs Working Group for Higgs boson searches Collaboration, 'Search for charged Higgs bosons: Preliminary combined results using LEP data collected at energies up to 209-GeV', arXiv:hep-ex/0107031. 
[32] LEP Higgs Working Group for Higgs boson searches Collaboration, R. Barate et al., 'Search for the standard model Higgs boson at LEP', Phys. Lett. B565 (2003) 61 [arXiv:hep-ex/0306033];

OPAL Collaboration, G. Abbiendi et al., 'Flavour independent search for Higgs bosons decaying into hadronic final states in e+ e- collisions at LEP', arXiv:hepex/0312042; 'Decay-mode independent searches for new scalar bosons with the OPAL detector at LEP', Eur. Phys. J. C27 (2003) 311 [arXiv:hep-ex/0206022]; LEP Higgs Working Group for Higgs boson searches Collaboration, 'Flavor independent search for hadronically decaying neutral Higgs bosons at LEP', arXiv:hepex/0107034; 'Searches for Higgs Bosons Decaying into Photons: Combined Results from the LEP Experiments' LHWG Note/2002-02; 'Searches for invisible Higgs bosons: Preliminary combined results using LEP data collected at energies up to 209-GeV', arXiv:hep-ex/0107032;

ALEPH Collaboration, D. Buskulic et al., 'Search for a nonminimal Higgs boson produced in the reaction e+ e- $\rightarrow$ h Z ${ }^{*}$, Phys. Lett. B313 (1993) 312;

DELPHI Collaboration, 'Search for Neutral Higgs Bosons in Extended Models' CERN EP 2003-061 (Submitted to EPJ).

[33] G. Hiller, ' $b$-physics signals of the lightest CP-odd Higgs in the NMSSM at large tan $\beta$.', Phys. Rev. D70 (2004) 034018 [arXiv:hep-ph/0404220].

[34] S. Baek, Y. G. Kim and P. Ko, 'Neutralino dark matter scattering and $B_{s} \rightarrow \mu^{+} \mu^{-}$ in SUSY models', arXiv:hep-ph/0406033.

[35] D.G. Cerdeño, E. Gabrielli, D. E. López-Fogliani, C. Muñoz and A. M. Teixeira, in preparation.

[36] For a review, see G. Jungman, M. Kamionkowski and K. Griest, 'Supersymmetric dark matter', Phys. Rept. 267 (1996) 195 [arXiv:hep-ph/9506380].

[37] For a summary, see also: U. Chattopadhyay, T. Ibrahim and P. Nath, 'Effects of $\mathrm{CP}$ violation on event rates in the direct detection of dark matter', Phys. Rev. D60 (1999) 063505 [arXiv:hep-ph/9811362];

T. Falk, A. Ferstl and K. A. Olive, 'Variations of the neutralino elastic crosssection with CP violating phases', Astropart. Phys. 13 (2000) 301 [arXiv:hep$\mathrm{ph} / 9908311]$.

[38] J. R. Ellis, A. Ferstl and K. A. Olive, 'Re-evaluation of the elastic scattering of supersymmetric dark matter', Phys. Lett. B481 (2000) 304 [arXiv:hep-ph/0001005]. 
[39] See e.g., Y.G. Kim, T. Nihei, L. Roszkowski and R. Ruiz de Austri, 'Upper and lower limits on neutralino WIMP mass and spin-independent scattering cross section, and impact of new $(g-2)_{\mu}$ measurement', J. High Energy Phys. 10 (2004) 015 [arXiv:hep-ph/0208069].

[40] See e.g., D. G. Cerdeño and C. Muñoz, 'Neutralino dark matter in supergravity theories with non-universal scalar and gaugino masses', J. High Energy Phys. 07 (2002) 024 [arXiv:hep-ph/0405057], and references therein.

[41] D. G. Cerdeño, M. Gómez, C. Hugonie, D. E. López-Fogliani, C. Muñoz and A. M. Teixeira, in preparation.

[42] WMAP Collaboration, C.L. Bennett et al., 'First year Wilkinson microwave anisotropy probe (WMAP) observations: preliminary maps and basic results', Astrophys. J. Suppl. 148 (2003) 1 [arXiv:astro-ph/0302207];

D.N. Spergel et al., 'First year Wilkinson microwave anisotropy probe (WMAP) observations: determination of cosmological parameters', Astrophys. J. Suppl. 148 (2003) 175 [arXiv:astro-ph/0302209].

[43] G. Belanger and C. Hugonie, in preparation. 